\title{
Gap Junction Channels and Cardiac Impulse Propagation
}

\author{
Thomas Desplantez · Emmanuel Dupont • \\ Nicholas J. Severs $\cdot$ Robert Weingart
}

Received: 14 May 2007/ Accepted: 15 May 2007/Published online: 28 July 2007

(C) Springer Science+Business Media, LLC 2007

\begin{abstract}
The role of gap junction channels on cardiac impulse propagation is complex. This review focuses on the differential expression of connexins in the heart and the biophysical properties of gap junction channels under normal and disease conditions. Structural determinants of impulse propagation have been gained from biochemical and immunocytochemical studies performed on tissue extracts and intact cardiac tissue. These have defined the distinctive connexin coexpression patterns and relative levels in different cardiac tissues. Functional determinants of impulse propagation have emerged from electrophysiological experiments carried out on cell pairs. The static properties (channel number and conductance) limit the current flow between adjacent cardiomyocytes and thus set the basic conduction velocity. The dynamic properties (voltage-sensitive gating and kinetics of channels) are responsible for a modulation of the conduction velocity during propagated action potentials. The effect is moderate and depends on the type of $\mathrm{Cx}$ and channel. For homomeric-homotypic channels, the influence is small to medium; for homomeric-heterotypic channels, it is medium to strong. Since no data are currently available on heteromeric channels, their influence on impulse propagation is speculative. The modulation by gap junction channels is most prominent in tissues at the boundaries between cardiac tissues such as sinoatrial node-atrial muscle, atrioventricular node-His bundle, His bundle-bundle branch and Pur-
\end{abstract}

T. Desplantez $\cdot$ R. Weingart ( $\square)$

Institute of Physiology, University of Bern, Bühlplatz 5, CH-

3012, Bern, Switzerland

e-mail: weingart@pyl.unibe.ch

E. Dupont · N. J. Severs

Cardiac Medicine, National Heart and Lung Institute, Imperial

College London, London SW3 6LY, UK kinje fibers-ventricular muscle. The data predict facilitation of orthodromic propagation.

Keywords Heart - Gap junction · Connexin - Impulse propagation · Disease

\section{Introduction}

Impulse propagation in cardiac tissue is determined by source and sink factors. The source factors involve excitability of cell membranes brought about by excitatory inward currents; the sink factors include passive electrical properties of cells and tissue architecture (cell geometry, cell connectivity). A modification of any one of these elements is predicted to alter conduction velocity in the heart.

Since the pioneering work by Weidmann $(1952,1966)$, it has been known that cardiac cells are functionally connected with each other. His elegant experiments demonstrated that small ions are able to move from cell to cell via specialized cell-to-cell interaction called "intercalated disks," driven by either electric fields or concentration gradients, suggesting that cardiac cells are coupled electrically and/or diffusionally. Subsequently, it was shown that the low-resistance intercellular pathways consist of aqueous transcellular channels. These channels are typically aggregated in the form of gap-junctional (GJ) plaques (see Fig. 1).

Subsequently, enormous progress has been made in the field of GJ research, structurally and functionally. The realization grew that GJ channels (GJCs) are not merely a curiosity restricted to excitable tissues such as mammalian heart muscle (Weidmann, 1967; lecture delivered on Sept. $23,1965)$ or insect salivary glands (Loewenstein et al., 1965; reviewed by Loewenstein, 1981). Today, we know 


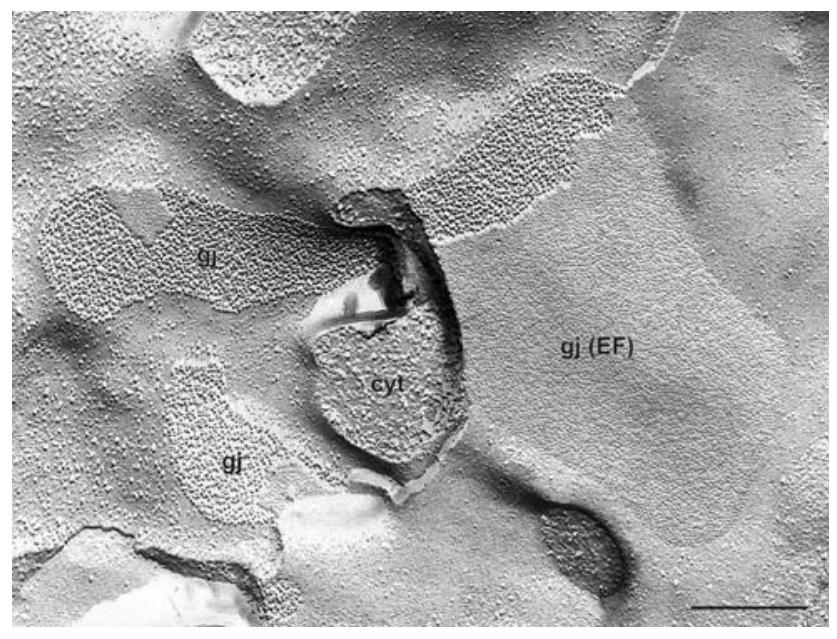

Fig. 1 Freeze-fracture electron micrograph illustrating the typical structure of gap junctions $(g j)$ at the cardiac intercalated disk. The gap junctions appear as clusters of particles, corresponding to $\mathrm{HCs}$, in the membrane plane. This characteristic appearance is found on the $\mathrm{P}$ face of the membrane (the fracture face of the half-membrane sheet left attached to the protoplasm). In the upper of the two plasma membrane contributing to the gap junction (seen in E-faceview; $E F$ ), arrays of pits are seen. These are the sites at which the HCs havebeen fractured away when the membrane is split by freeze fracture (cyt cytoplasm). Scale bar $=250 \mathrm{~nm}$

that GJs are present in almost all cells of vertebrates, exceptions being circulating blood cells and adult skeletal muscle. Electron microscopists and structural biochemists have demonstrated that GJs are composed of aggregates of cell-cell channels, with each channel constructed from two hemichannels (HCs) and each $\mathrm{HC}$ comprising six subunits. Molecular biologists have identified the component proteins of GJCs as connexins (Cxs) and discovered that there are at least 20 different mammalian Cxs (Willecke et al., 2002). Electrophysiologists have acquired a substantial body of knowledge on biophysical properties of GJs and GJCs over the last two decades. Cell-pair preparations, consisting of communication-deficient cell lines transfected with Cxs or Xenopus oocytes injected with Cx transcripts or cells freshly dissociated from tissues, turned out to be most useful. These approaches revealed that, in common with other membrane channels, GJCs exhibit electrical and chemical gating.

The aim of this review is to elaborate on the role of GJCs in the propagation of the cardiac impulse by integrating data gathered at the molecular and cellular levels into tissues of the intact heart. Special emphasis will be placed on the aspects of different types of GJCs.

\section{Propagation of the Cardiac Impulse}

The mechanism underlying impulse propagation in the heart is of great interest for clinicians and basic scientists.
This is because alterations in conduction velocity $(\theta)$ may contribute to cardiac arrhythmias. At the organ level, the cardiac action potential propagates over the entire heart via a well-established pathway. It is initiated in the sinoatrial (SA) node, spreads over the atria, is then directed through the atrioventricular (AV) node along the specialized ventricular conduction system and invades both ventricles to elicit a quasi-synchronous mechanical activation of the working myocardium. To separate the activation of the atria from the ventricles, the impulse slows down during its transit through the AV node.

At the cellular level, propagation of an action potential involves local circuit currents. Local circuits may be regarded as closed loops consisting of four discrete elements: (1) channels mediating the excitatory inward current carried by $\mathrm{Na}^{+}$or $\mathrm{Ca}^{2+}\left(I_{\mathrm{Na}}, I_{\mathrm{Ca}}\right)$; (2) intracellular longitudinal resistance, $r_{\mathrm{i}}\left(r_{\mathrm{i}}=r_{\mathrm{c}}+r_{\mathrm{j}} ; r_{\mathrm{c}}\right.$, resistance of cytoplasm; $r_{\mathrm{j}}$, resistance of GJ); (3) capacitance of the nonjunctional membrane, $c_{\mathrm{m}}$; and (4) extracellular longitudinal resistance, $r_{\mathrm{o}}$. In essence, the conduction velocity of an action potential $(\theta)$ is determined by these four elements. Element 1 is the source factor. It exerts its effect via maximal upstroke velocity of action potentials, $d V / d t_{\max }$. Elements $2-4$ are sink factors and consume electric charge. Elements 1,2 and 4 are variable and hence of particular interest. The role of 1 is well established. The importance of 2 and 4 was recognized when it was discovered that $r_{\mathrm{i}}$ and $r_{\mathrm{o}}$ can be modified under certain conditions. However, most relevant in the present context is the role of 2. To a first approximation, $\theta^{2}$ is proportional to $d V / d t_{\max }$ and inversely proportional to $\left(r_{\mathrm{o}}+r_{\mathrm{i}}\right)$. The space constant, $\lambda$, is a useful parameter to describe the tendency for current to spread between adjacent cells. In a linear cable, $\lambda^{2}$ correspond to $r_{\mathrm{m}} /\left(r_{\mathrm{o}}+r_{\mathrm{i}}\right)$. For a more elaborate treatment of the concept of cardiac impulse propagation, see, e.g., Kléber \& Rudy (2004).

\section{Cx Expression in the Normal Adult Heart}

Multiple Cxs are coexpressed in the heart. Three principal Cxs are found in cardiac myocytes, Cx43, Cx40 and Cx45. $\mathrm{Cx} 43$ predominates in the heart as a whole but is typically coexpressed in characteristic combinations and relative quantities with $\mathrm{Cx} 40$ and/or Cx45 in a chamber-related and myocyte type-specific manner (Van Kempen et al., 1995; Vozzi et al., 1999; Severs et al., 2004a, 2006). Recently, an additional $\mathrm{Cx}, \mathrm{Cx} 30.2$, has been reported in the mouse $\mathrm{AV}$ node (Kreuzberg et al., 2006). In addition, a few further Cx types have been noted in past studies of cardiac tissue, but these remain unconfirmed. A summary of the typical $\mathrm{Cx}$ expression patterns of the normal adult human heart is shown in Table 1. 
Table 1 Relative expression levels of Cxs in human cardiac tissues (estimated expression levels are based on immunoconfocal microscopy)

\begin{tabular}{llllllll}
\hline Cx isoform & SA node & Atrium & Compact AV node & His bundle & Bundle branch & Purkinje fiber & Ventricle \\
\hline 40 & - & ++++ & - & +++ & ++++ & + & +++ \\
43 & - & ++++ & - & ++ & +++ & +++ & +++ \\
45 & +++ & + & +++ & ++ & + & + & + \\
\hline
\end{tabular}

Scale: ++++, very abundant; \pm , barely detectable. Quantities confirmed by Western and Northern blots are indicated in bold. Cx40 and Cx43 in atria are expressed at similar levels relative to each other. Note that along the conduction system and within the AV node, the patterns of coexpression vary according to species

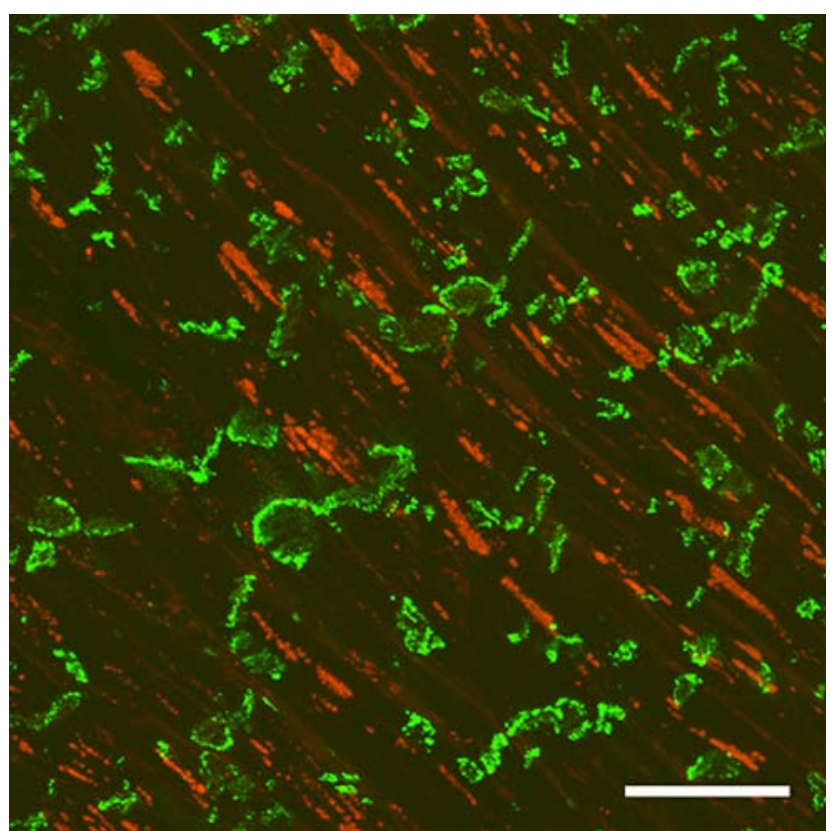

Fig. 2 Immunostaining for $\mathrm{Cx} 43$ in human ventricle. GJs (labeled in green) clearly delineate the steplike intercalated disks, with larger, brighter gap junctions at the disk periphery. The red/orange fluorescence is generated by lipofuscin, a strongly autofluorescent material that is deposited centrally on each side of the nuclei. Scale bar $=50 \mathrm{~mm}$

Myocytes are not uniform cells but vary in morphology according to their location in the heart. The working myocytes of the ventricle and atrium are rod-shaped cells which are extensively interconnected by clusters of GJs (see Fig. 2). The GJs are organized, together with fasciae adherentes junctions and desmosomes, at the intercalated disks (Severs, 1990) (see Fig. 3). The disks join abutting myocytes in a step-like structure so that the myofibrils of consecutive cells are mechanically joined in series at the fasciae adherentes junctions, i.e., in the vertical segments of disk steps. The GJs are located predominantly in the longitudinal segments of the steps. Desmosomes, which link to the intermediate filament (desmin) cytoskeleton, are found in association with both the GJs and the fasciae adherentes. Ventricular myocytes are significantly larger in diameter than atrial myocytes and have particularly extensive, well-developed intercalated disks. Larger GJs are typically arranged around the periphery of the intercalated disk, with smaller junctions in the interior region (Hoyt, Cohen \& Saffitz, 1989; Severs, 1989b; Gourdie, Green \& Severs, 1991). GJ organization together with features of tissue architecture such as the size and shape of the cells combine to determine the normal anisotropic pattern of impulse spread in healthy myocardium.

Cx43 is abundantly expressed in ventricular myocyte GJs, often coexpressed with trace amounts of $\mathrm{Cx} 45$. Atrial myocytes express the same quantity of $\mathrm{Cx} 43$ as ventricular myocytes but in addition have approximately equal amounts $\mathrm{Cx} 40$ together with slightly higher levels of $\mathrm{Cx} 45$ than are found in the ventricle (Vozzi et al., 1999). Typically, the coexpressed Cxs are found as mixtures within the same GJ plaque (Severs et al., 2001).

Apart from myocytes whose function is contraction, the myocytes specialized to generate and conduct the impulse have distinctive morphological and $\mathrm{Cx}$ expression features (Severs, 1989a). Myocytes of the SA and AV nodes have small, dispersed GJs composed of Cx45 (Coppen et al., 1999a; Coppen, Severs \& Gourdie, 1999b). In the mouse, these cells reportedly also express Cx30.2 (Kreuzberg et al., 2006). In vitro biophysical studies show that both these Cxs form low-conductance channels (Van Veen, van Rijen \& Jongsma, 2000; Rackauskas et al., 2007). The size, arrangements and $\mathrm{Cx}$ composition of the nodal GJs are indicative of low coupling, which contributes to slowing of impulse propagation. In this way, the AV node ensures the sequential contraction of the cardiac chambers.

In the SA node, a connective tissue layer separates the node from the surrounding atrial myocardium except at a restricted zone of $\mathrm{Cx} 45 / \mathrm{Cx} 43$ coexpression at the nodal/ crista terminalis border. These features may contribute to the ability of the node (small source) to drive the large mass of surrounding atrial tissue (large sink) while remaining protected from its hyperpolarizing influence. The zone of $\mathrm{Cx} 45 / \mathrm{Cx} 43$ coexpression has been hypothesized as the exit route for the impulse into the atrial tissue (Coppen et al., 1999a).

Three-dimensional reconstructions of the rabbit $\mathrm{AV}$ node reveal distinctive compartmentalized $\mathrm{Cx}$ expression 
Fig. 3 Thin-section electron micrograph illustrating structure of the intercalated disk between two rat ventricular myocytes. Fasciae adherentes junctions (fa) mechanically couple the myofibrils (contractile apparatus) of the adjacent cells; desmosomes $(d)$ serve as anchor points for the desmin cytoskeleton. GJs $(g j)$ are situated in the membrane segments between the fasciae adherentes. Scale bar $=1 \mu \mathrm{m}$

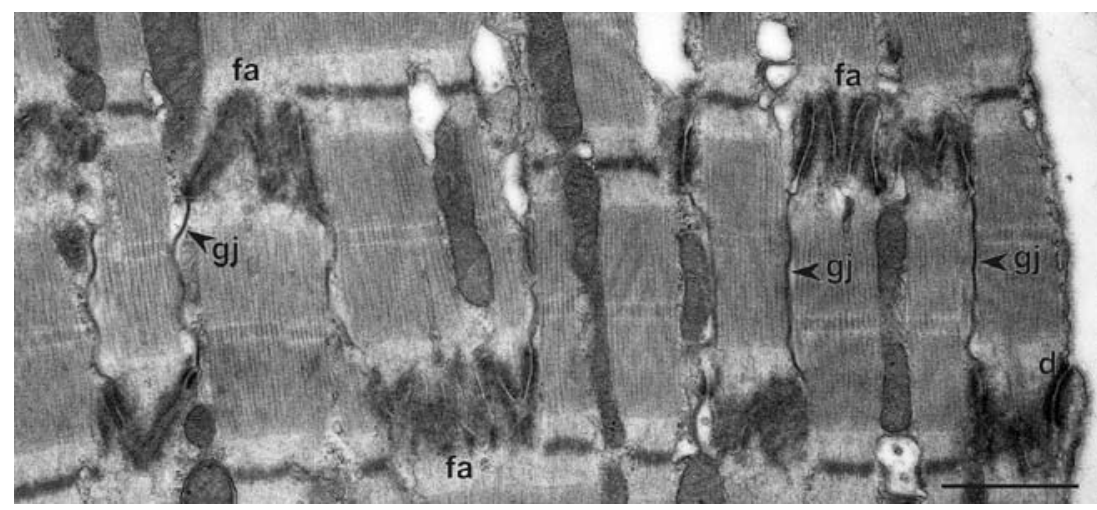

patterns, with the compact node and transitional cells predominantly expressing Cx45 and the His bundle, lower nodal cells and posterior nodal extension coexpressing Cx45 with Cx43 (Ko et al., 2004). Downstream from the His bundle, the conduction system myocytes typically express high levels of Cx40 (Gourdie et al., 1993; Miquerol et al., 2004). In rodents, $C x 45$ is coexpressed with $\mathrm{Cx} 40$ in a central zone of the bundle branches and Purkinje fibers, enveloped by an outer zone in which only $\mathrm{Cx} 45$ is found (Coppen et al., 1999b). Apart from Cx40 and Cx45, distal parts of the conduction system also express $\mathrm{Cx} 43$ (Gourdie et al., 1993; Van Kempen et al., 1995).

Although different mammalian species typically share the features of $\mathrm{Cx}$ expression described above, there are some notable exceptions, e.g., lack of $\mathrm{Cx} 40$ in rat atrial muscle and guinea pig conduction system (Gros et al., 1994; Van Kempen et al., 1995). Species variations in patterns of coexpression within the AV node between large and small mammals have also been reported and may be related to the greater need for impulse delay in the latter (Coppen \& Severs, 2002). Thus, it should not be assumed that the presence of $\mathrm{Cx} 30.2$ in the mouse AV node necessarily implies that the human orthologue, $\mathrm{Cx} 31.9$, is required in the same location. Ongoing work suggests that the spatial patterns of $\mathrm{Cx}$ coexpression along the course of the human conduction system may differ in detail from those of the mouse and rat (Kaba et al., 2003). Defining species-specific patterns of cardiac $\mathrm{Cx}$ expression is a critical factor in interpreting the relevance of functional data obtained from studies on cells derived from transgenic mice and transfected cell models to the human heart in health and disease.

\section{Cx Expression in Heart Disease}

Acquired adult heart disease in which there is an arrhythmic tendency is marked by remodeling of GJs and $\mathrm{Cx}$ expression. Two overlapping forms of remodeling may occur, (1) structural remodeling, which involves changes in

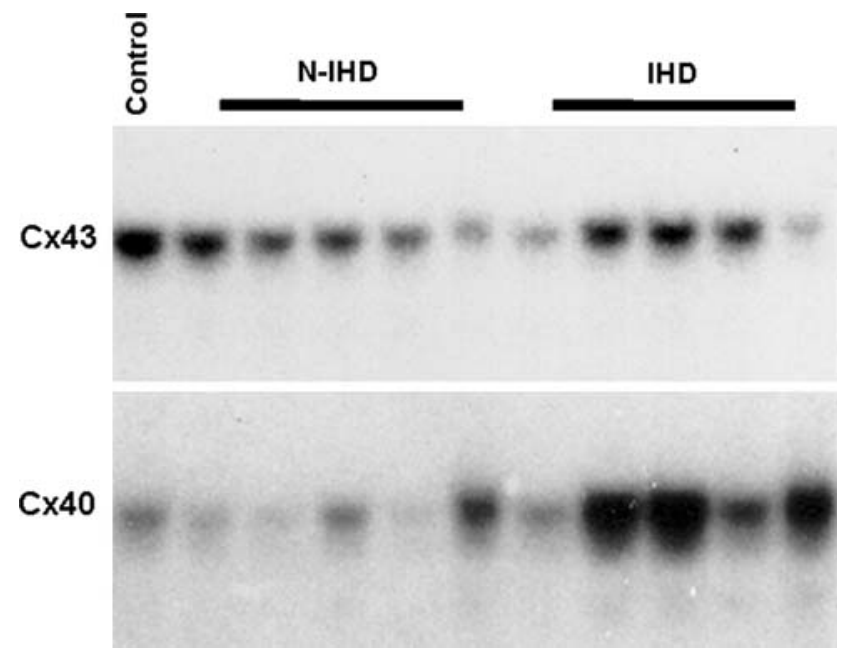

Fig. 4 Northern blot analysis of $\mathrm{Cx} 43$ and $\mathrm{Cx} 40$ in the left ventricle of patients with end-stage heart failure due to idiopathic dilated cardiomyopathy (nonischemic heart disease, $N-I H D$, or ischemic heart disease, $I H D)$. Note that Cx43 mRNA is markedly downregulated, more so in some samples than others. The loss of $\mathrm{Cx} 43$ can, in some instances, exceed 90\%. In addition, $\mathrm{Cx} 40$ is strongly upregulated (up to four or five times) in the ischemic category. Note that these hybridizations are done on the same membrane

the arrangement or organization of GJs (Smith et al., 1991), and (2) remodeling of $\mathrm{Cx}$ expression, in which the amount and/or types of $\mathrm{Cx}$ expressed are altered (Severs et al., 2006).

For details of structural remodeling, the reader is referred to other reviews (Severs et al., 2004a,b, 2005, 2006). With regard to alterations in $\mathrm{Cx}$ expression, the most widely documented change is downregulation of $\mathrm{Cx} 43$ in the ventricle of patients with end-stage congestive heart failure (see Fig. 4). This change occurs regardless of the primary cause of the condition (e.g., idiopathic dilated cardiomyopathy, ischemic cardiomyopathy, valvular aortic stenosis or other etiologies) (Dupont et al., 2001; Kitamura et al., 2002; Kostin et al., 2003, 2004; Yamada et al., 2003). The reduction in ventricular $\mathrm{Cx} 43$ appears to develop progressively during the course of disease, as suggested by 
Fig. 5 Homomeric-homotypic GJs. Dependence of GJ conductance on junctional potential for $\mathrm{Cx} 40-\mathrm{Cx} 40$ (a), $\mathrm{Cx} 43-\mathrm{Cx} 43$ (b) and $\mathrm{Cx} 45-\mathrm{Cx} 45$ (c) channels. Upper panels: Superimposed records of GJ currents elicited by a standard pulse protocol. Lower panels: Plots of normalized instantaneous $(\bigcirc)$ and steadystate $(\bullet)$ conductance as functions of $V_{\mathrm{j}}$. Sources: Cx40Cx40 (T. Desplantez, D. Halliday, E. Dupont \& R. Weingart, unpublished data), Cx43-Cx43, Cx45-Cx45 (Desplantez et al., 2004)
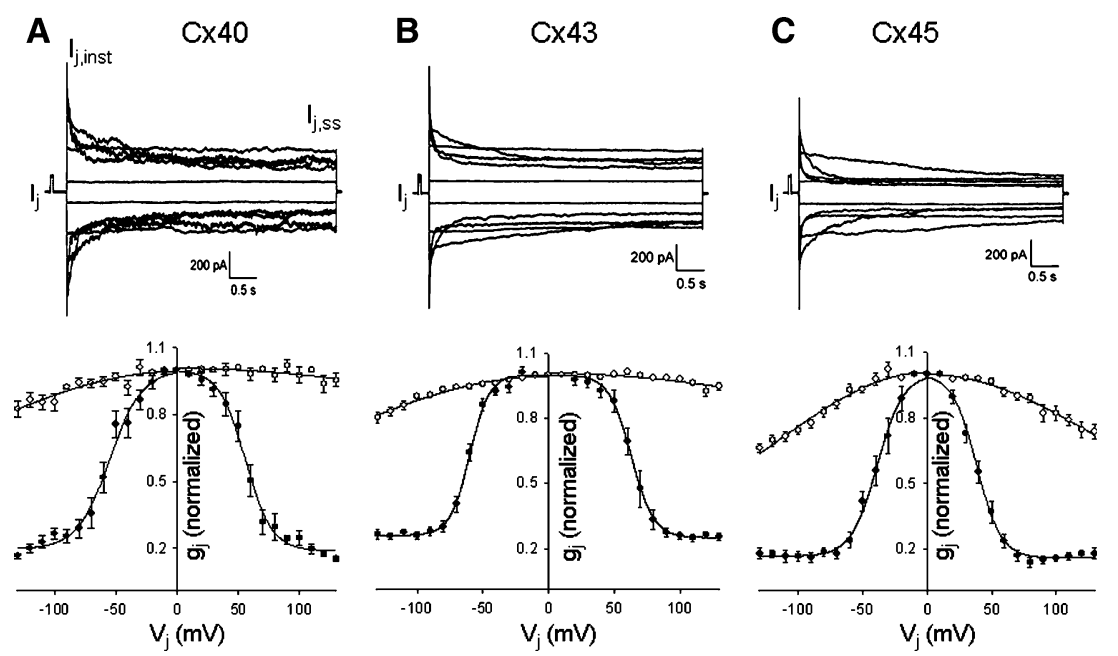

the pattern of change observed in pressure-overloaded hearts with valvular aortic stenosis classified according to ejection fraction (Kostin et al., 2004) and the finding of reduced $\mathrm{Cx} 43$ in the nonfailing ventricles of coronary artery bypass patients (Peters et al., 1993).

Alongside reduced $\mathrm{Cx} 43$ levels, increased expression of Cx45 (Yamada et al., 2003) and Cx40 (Dupont et al., 2001) have been reported in the failing human ventricle (see Fig. 4). Increased levels of $\mathrm{Cx} 40$ transcript have been detected in the ventricles of patients with congestive heart failure due to ischemic cardiomyopathy but not that due to idiopathic dilated cardiomyopathy (Dupont et al., 2001). This elevated Cx40 expression correlates with an increased depth of Cx40-expressing myocytes from the endocardial surface, in the location corresponding and adjacent to Purkinje myocytes. Thus, the Cx coexpression patterns of the diseased human ventricle involve a generalized increase in the ratio of $\mathrm{Cx} 45: \mathrm{Cx} 43$ and, in ischemic heart disease, a localized increase in $\mathrm{Cx} 40: \mathrm{Cx} 43$.

In human ventricle, $\mathrm{Cx} 43$ and $\mathrm{Cx} 45$ colocalize to the same GJ plaques (Vozzi et al., 1999; Yamada et al., 2003) as do $\mathrm{Cx}_{40}$ and $\mathrm{Cx} 43$ in Purkinje fibers (Severs et al., 2001). The potentially profound effects of coexpression of Cx43 and $\mathrm{Cx} 40$ are highlighted by studies using cultured atrial cells from homozygous and heterozygous knockout mice. In these coexpressing cells, increasing the ratio of $\mathrm{Cx} 40$ to $\mathrm{Cx} 43$ has the counterintuitive effect of reducing propagation velocity (Beauchamp et al., 2006). Thus, it might be speculated that increased endocardial expression of $\mathrm{Cx} 40$ in human ischemic heart disease might change the pattern of activation at Purkinje/working ventricle myocyte junctions.

\section{Properties of GJCs}

As mentioned at the outset, GJs are assemblies of intercellular channels, each of which consists of two HCs. The six subunits originally identified in each HC represent six Cx molecules, which span the full depth of the membrane. The specific Cx type or mix of $\mathrm{Cx}$ types within the HC is thought to be a key determinant of the functional properties of the GJCs (reviewed by Harris, 2001). The term "homomeric" is used to describe an HC composed of a single Cx type; correspondingly, "heteromeric" is used to designate an $\mathrm{HC}$ constructed from two or more $\mathrm{Cx}$ types. The channel as a whole may be "homotypic" (i.e., made from matching HCs) or "heterotypic" (nonmatching HCs). The presence of multiple Cxs within the same channel opens the possibility of a wide range of different heteromeric HCs and GJCs. A major unanswered question is how these translate into differentiation of electrophysiological (and diffusional) properties.

\section{Homomeric-Homotypic GJCs}

Figure 5 illustrates the behavior of homomeric-homotypic GJCs made of Cx40, Cx43 and Cx45. These channels have been the subject of manifold studies (e.g., Bukauskas et al., 1995; Moreno et al., 1995; Barrio et al., 1997; Valiunas et al., 2000; Desplantez et al., 2004; reviewed by Harris, 2001). The data depicted were gained from pairs of transfected cells by means of the dual voltage-clamp technique. The upper panels show GJ currents, $I_{\mathrm{j}}$, recorded when one cell of a cell pair was depolarized/hyperpolarized (downward/upward signals) to establish a negative/positive transjunction voltage, $V_{\mathrm{j}}$, respectively. Comparison within panels reveals that $I_{\mathrm{j}}$ grows larger and inactivates faster with increasing $V_{\mathrm{j}}$, giving rise to steady-state and instantaneous components, $I_{\mathrm{j}, \mathrm{ss}}$ and $I_{\mathrm{j}, \text { inst }}$, respectively.

The lower panels show the analysis of $I_{\mathrm{j}}$ records in terms of normalized GJ conductance, $g_{\mathrm{j}}=I_{\mathrm{j}} / V_{\mathrm{j}}\left(g_{\mathrm{j}}=1 / r_{\mathrm{j}}\right)$. The smooth curve corresponds to the best fit of data to the function $g_{\mathrm{j}, \text { inst }}=f\left(V_{\mathrm{j}}\right)$ (see equation 1 in Desplantez et al., 2004). It reflects the predicted nonohmic behavior of HCs 
Table 2 Summary of data obtained from multichannel and single-channel currents of homomeric-homotypic channels

\begin{tabular}{llllll}
\hline Channel type & $V_{\mathrm{j}, 0}(\mathrm{mV})$ & $\mathrm{Z}$ & $g_{\mathrm{j}, \text { min }}$ & $\gamma_{\mathrm{j}, \text { main }}(\mathrm{pS})$ & 162 \\
\hline Cx40-Cx40 & $-55 / 56$ & $-1.8 / 2.3$ & $0.20 / 0.19$ & 61 & 28 \\
Cx43-Cx43 & $-61 / 63$ & $-3.4 / 2.9$ & $0.26 / 0.25$ & 32 & 12 \\
Cx45-Cx45 & $-39 / 39$ & $-2.5 / 2.7$ & $0.17 / 0.16$ & $60 / 100$ & 12 \\
Cx40-Cx43 & $-82 /-$ & $-1.9 /-$ & $0.31 /-$ & $30 / 40$ & $14 / 20$ \\
Cx40-Cx45 & -150 & $-/ 3.3$ & $-/ 0.06$ & 40 & 11 \\
Cx43-Cx45 & $-12 / 134$ & $-2.1 / 0.6$ & $0.05 /-$ & $\mathrm{pS})$ \\
\hline
\end{tabular}

For explanations, see text. Experimental conditions: transfected cells, pipette solution $\left(\mathrm{K}^{+}\right.$aspartate $\left.{ }^{-}\right)$, room temperature. Sources (multichannel/ single channel): Cx40-Cx40 (Desplantez, unpublished; Bukauskas et al., 1995), Cx43-Cx43 (Desplantez et al., 2004; Valiunas et al., 1997), Cx45-Cx45 (Desplantez et al., 2004; T. Desplantez \& R. Weingart, unpublished), Cx40-Cx43 (Valiunas et al., 2000), Cx40-Cx45 (Valiunas et al., 2000; V. Valiunas, unpublished). Cx43-Cx45 (Desplantez et al., 2004; T. Desplantez \& R. Weingart, unpublished)

(Vogel \& Weingart, 1998, 2002). The bell-shaped curve represents the best fit of data to the function $g_{\mathrm{j}, \mathrm{ss}}=f\left(V_{\mathrm{j}}\right)$ (see equation 2 in Desplantez at al., 2004). It reflects the transition of GJCs from the open to the residual state. At $V_{\mathrm{j}}=0 \mathrm{~m} V$, the channels are usually in the open state, at large $V_{\mathrm{j}}$ in the residual state. $V_{\mathrm{j}}$ controls the probability of the channels to be in these states. For reasons of symmetry, only one $\mathrm{HC}$ of such a GJC is gating at a given $V_{\mathrm{j}}$ polarity. Hence, in the residual state one $\mathrm{HC}$ is residing in a lowconductance configuration, the other in a high-conductance configuration (Vogel \& Weingart, 1998, 2002). Comparison among panels indicates that the $V_{\mathrm{j}}$ sensitivity of gating is Cx-specific; i.e., it follows the sequence $\mathrm{Cx} 45>\mathrm{Cx} 40>$ Cx43.

Table 2 summarizes the Boltzmann parameters for the three kinds of channels. They reflect the $V_{\mathrm{j}}$ sensitivity of gating. $V_{\mathrm{j}, 0}$ is the voltage at which $g_{\mathrm{j}, \mathrm{ss}} / g_{\mathrm{j} \text {,inst }}$ is half-maximally inactivated, $z$ is the number of positive charges moving through the electric field applied and $g_{\mathrm{j}, \min }$ is the minimal $g_{\mathrm{j}, \mathrm{ss}}$ at large $V_{\mathrm{j}}$. The smaller $V_{\mathrm{j}, 0}$, the larger is the $V_{\mathrm{j}}$ sensitivity of $g_{\mathrm{j}, \mathrm{ss}}$; the larger $z$, the steeper is the maximal slope of the $V_{\mathrm{j}}$-dependent decay of $g_{\mathrm{j}, \mathrm{ss}}$.

Cells expressing Cx40, Cx43 or Cx45 have also been used to explore the properties of single GJCs. Data were obtained from de novo formed cell pairs (Bukauskas et al., 1995) and preformed cell pairs, weakly coupled or normally coupled and exposed to submaximal doses of an uncoupling agent, e.g., $n$-heptanol. The channels have two

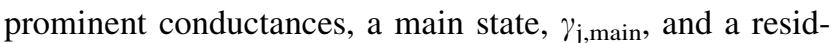
ual state, $\gamma_{\mathrm{j}, \text { residual }}$. The transitions between states are fast (1-2 ms). Table 2 also summarizes the conductance data, which follow the sequence $\mathrm{Cx} 40>\mathrm{Cx} 43>\mathrm{Cx} 45$. The residual state explains the incomplete decay of $g_{\mathrm{j}, \mathrm{ss}}$ at large $V_{\mathrm{j}}$ (see Fig. 3). Measurements at different $V_{\mathrm{j}}$ show that $\gamma_{\mathrm{j} \text {,main }}$ is slightly $V_{\mathrm{j}}$-sensitive (e.g., Valiunas \& Weingart, 2000; Valiunas, 2002) in agreement with the slight curvature of $g_{\mathrm{j} \text {,inst }}=f\left(V_{\mathrm{j}}\right)$ (see Fig. 3).

A more comprehensive description of the behavior of GJCs includes kinetic analyses of $I_{\mathrm{j}}$. Currents through GJCs undergo a time-dependent inactivation during $V_{\mathrm{j}}$ pulses and a time-dependent recovery from inactivation thereafter. With regard to inactivation, $I_{\mathrm{j}}$ remains constant at small $V_{\mathrm{j}}$. At intermediate $V_{\mathrm{j}}$, it inactivates slowly. The decay follows an exponential, giving rise to a time constant, $\tau_{\mathrm{i}}$. At large $V_{\mathrm{j}}, I_{\mathrm{j}}$ inactivates rapidly. The decay follows a single exponential or the sum of two exponentials, leading to the time constants $\tau_{\mathrm{i} 1}$ or $\tau_{\mathrm{i} 1}$ and $\tau_{\mathrm{i} 2}$, respectively. Comparison of $\tau_{\mathrm{i}}$ values suggests that the kinetics of $I_{\mathrm{j}}$ inactivation follows the sequence $\mathrm{Cx} 40=\mathrm{Cx} 43<\mathrm{Cx} 45$.

In contrast to inactivation, data on recovery of $I_{\mathrm{j}}$ from inactivation are rudimentary (Veenstra, 1990; Wang et al., 1992). Systematic studies have recently been initiated using a dual-pulse protocol (T. Desplantez \& R. Weingart, unpublished data). A standard conditioning pulse was applied to maximally inactivate $I_{\mathrm{j}}$. At variable delays, a short test pulse was delivered to determine $I_{\mathrm{j}}$ during recovery. It turned out that $I_{\mathrm{j}}$ recovers exponentially with time, leading to a time constant, $\tau_{\mathrm{r}}$. Moreover, alteration of the amplitude of the test pulses reveals that $I_{\mathrm{j}}$ recovery speeds up with decreasing levels of $V_{\mathrm{j}}$. Hence, small values of $V_{\mathrm{j}}$ are accompanied by slow inactivation and fast recovery of $I_{\mathrm{j}}$ and large values of $V_{\mathrm{j}}$ are associated with fast inactivation and slow recovery.

Physiologically most relevant are the values of $\tau_{\mathrm{i}}$ at smaller $V_{\mathrm{j}}$ (see below, Role of the Junctional Voltage Gradient). Unfortunately, $I_{\mathrm{j}}$ records at these voltages do not show signs of inactivation. Conceivably, this is due to the small signal/noise ratios and/or inaccurate measurements of $I_{\mathrm{j}}$ owing to series resistance problems (Van Rijen et al., 1998).

A model by Harris, Spray \& Bennett (1981) proposes that changes in $g_{j}$ are governed by reversible first-order processes whose rate constant of channel opening $(\alpha)$ and closing $(\beta)$ depend on $V_{\mathrm{j}}$. The model is based on the finding that $I_{\mathrm{j}}$ decays and recovers exponentially with time. It predicts that $\tau_{\mathrm{i}}$ and $\tau_{\mathrm{r}}$ are identical at a given $V_{\mathrm{j}}$; i.e., both are elements of a unique function, $\tau=f\left(V_{\mathrm{j}}\right)$ with $\tau=1 /(\alpha$ $+\beta)$. The function is maximal at $V_{\mathrm{j}}$ around $30-40 \mathrm{~m} V$ and 
Fig. 6 Homomeric-heterotypic GJs. Dependence of GJ conductance on junctional potential for $\mathrm{Cx} 40-\mathrm{Cx} 43$ (a), $\mathrm{Cx} 40-\mathrm{Cx} 45$ (b) and $\mathrm{Cx} 43-\mathrm{Cx} 45$ channels (c). Upper panels: Superimposed records of GJ currents elicited by a standard pulse protocol. Lower panels: Plots of normalized instantaneous $(\bigcirc)$ and steadystate $(\bullet)$ conductance as functions of $V_{\mathrm{j}}$. Sources: Cx40Cx43, Cx40-Cx45 (Valiunas et al., 2000), Cx43-Cx45 (Desplantez et al., 2004)
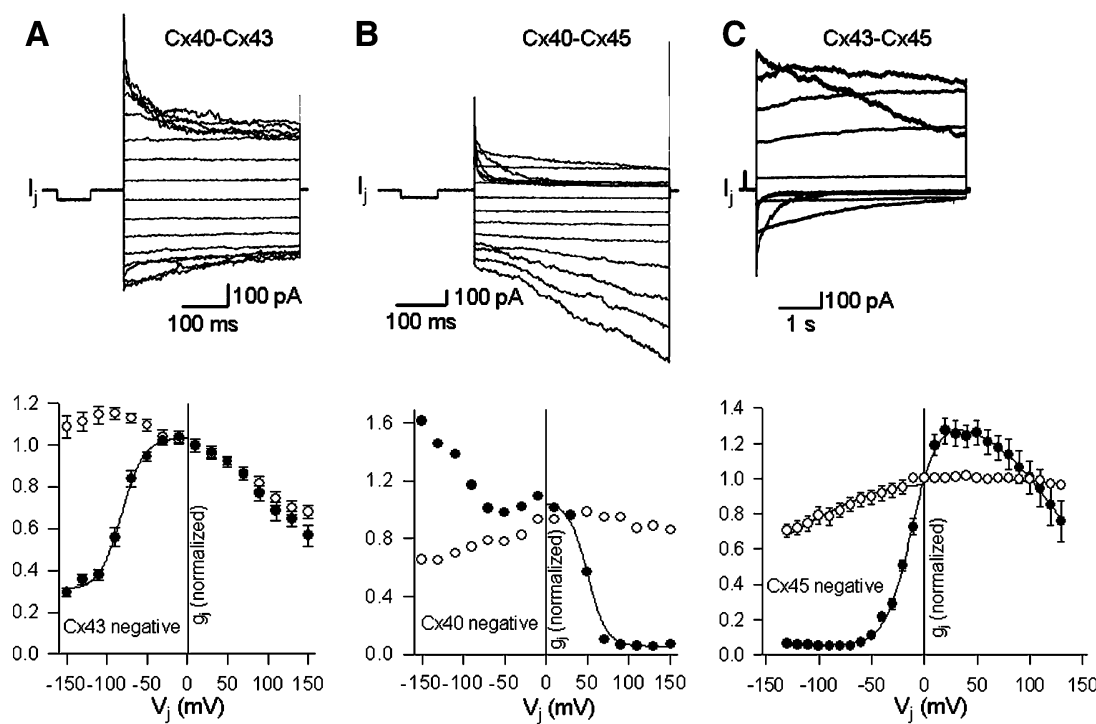

falls off sharply with smaller $V_{\mathrm{j}}$ and gradually with larger $V_{\mathrm{j}}$.

The kinetics of $I_{\mathrm{j}}$ inactivation and recovery from inactivation are crucial to appreciate the tissue-specific importance of GJs on impulse propagation. Hence, accurate measurements of $\tau$ for both processes are required, notably those covering the low $V_{\mathrm{j}}$ range. Besides furnishing missing data, this will help to verify if the vertebrate data are consistent with the model by Harris et al. (1981), originally derived from invertebrates.

While data on homomeric-homotypic channels provide useful insights into the electrophysiological correlates of individual Cxs, as discussed earlier, myocytes in vivo typically coexpress multiple Cxs. Whereas data on $\mathrm{Cx} 43$ channels are likely to give a fair representation of the properties of normal adult ventricular myocytes $(\mathrm{Cx} 45$ expression is minimal), extrapolation to other parts of the heart with complex coexpression patterns can be misleading. For example, because channels composed solely of Cx40 have a high $\gamma_{j \text {,main }}$, the presence of large amounts of Cx40 in the conduction system (distal to the AV node) is commonly supposed to facilitate rapid propagation of the impulse to the Purkinje fibers and into the ventricular myocardium. The reality is that, because in Purkinje fibers Cx40 is coexpressed with $\mathrm{Cx}_{4} 45$ and with $\mathrm{Cx} 43$, a large range of different molecular mixes is created, the properties of which cannot reliably be predicted from data on single Cxs expressed in vitro. Thus, analysis of the electrophysiological properties of heterotypic and heteromeric channels becomes imperative.

\section{Homomeric-Heterotypic Channels}

Figure 6 illustrates the properties of homomeric-heterotypic GJCs, Cx40-Cx43, Cx40-Cx45 and Cx43-Cx45.
Several groups have been involved in such studies (Cx40Cx43: Valiunas, Weingart \& Brink, 2000; Cottrell, Wu \& Burt, 2002; Cx40-Cx45: Valiunas et al., 2000; Cx43-Cx45: Valiunas et al., 2000, Elenes et al., 2001, Desplantez et al., 2004; reviewed by Harris, 2001). The upper panels show $I_{\mathrm{j}}$ records elicited by different $V_{\mathrm{j}}$ pulses. For each kind of cell pair, the size and time course of $I_{\mathrm{j}}$ are sensitive to the value and polarity of $V_{\mathrm{j}}$. In the case of $\mathrm{Cx} 40-\mathrm{Cx} 43, I_{\mathrm{j}}$ is larger and exhibits a more prominent inactivation when cell $\mathrm{Cx} 43$ is negative inside (upward signals). In the case of $\mathrm{Cx} 40$ $\mathrm{Cx} 45, I_{\mathrm{j}}$ is smaller and inactivates when cell $\mathrm{Cx} 40$ is positive inside (upward signals) and activates when it is negative inside (downward signals). In the case of $\mathrm{Cx} 43$ $\mathrm{Cx} 45, I_{\mathrm{j}}$ is smaller and inactivates when cell $\mathrm{Cx} 45$ is negative inside (downward signals).

The lower panels show the analysis of data plotting the normalized $g_{\mathrm{j}, \text { inst }}$ and $g_{\mathrm{j}, \mathrm{ss}} v s$. $V_{\mathrm{j}}$. For each type of cell pair, both relationships are asymmetrical. In the case of $\mathrm{Cx} 40$ $\mathrm{Cx} 43, g_{\mathrm{j}, \text { inst }}$ increases transiently with negative $V_{\mathrm{j}}$ and decreases gradually with positive $V_{\mathrm{j}}$, reflecting the $V_{\mathrm{j}}$ sensitivity of $\gamma_{\mathrm{j}, \text { main }}$. However, $g_{\mathrm{j}, \mathrm{ss}}$ decreases prominently with negative $V_{\mathrm{j}}$ and moderately with positive $V_{\mathrm{j}}$. The former follows a sigmoidal pattern and thus represents $V_{\mathrm{j}}$ gating; the latter follows $g_{\mathrm{j}, \text { inst }}=f\left(V_{\mathrm{j}}\right)$ and hence resembles the behavior of $\gamma_{\mathrm{j}, \text { main }}$. This suggests that $\mathrm{Cx} 40$ is gating with positive and $\mathrm{Cx}_{4} 43$ with negative polarity. In the case of Cx40-Cx $45, g_{\mathrm{j}, \text { inst }}$ decreases moderately with negative $V_{\mathrm{j}}$ and slightly with positive $V_{\mathrm{j}}$. Again, this can be explained by the properties of $\gamma_{\mathrm{j} \text {,main }}$. However, $g_{\mathrm{j}, \text { ss }}$ prominently increases with negative $V_{\mathrm{j}}$ and decreases with positive $V_{\mathrm{j}}$. The former increases beyond $g_{\mathrm{j}, \text { inst }}$ and hence reflects opening of previously closed channels; the latter follows a sigmoidal pattern and thus reflects $V_{\mathrm{j}}$ gating. This suggests that $\mathrm{Cx} 40$ is gating with positive and $\mathrm{Cx} 45$ with negative polarity. In the case of $\mathrm{Cx} 43-\mathrm{Cx} 45, g_{\mathrm{j}, \text { inst }}$ decreases 
moderately with negative $V_{\mathrm{j}}$ and barely with positive $V_{\mathrm{j}}$, representing the behavior of $\gamma_{\mathrm{j} \text {,main }}$. However, $g_{\mathrm{j}, \mathrm{ss}}$ decreases prominently with negative $V_{\mathrm{j}}$ and increases transiently with positive $V_{\mathrm{j}}$. The former follows a sigmoidal pattern and hence reflects $V_{\mathrm{j}}$ gating; the latter increases transiently beyond $g_{\mathrm{j}, \text { inst }}$ and thus reflects opening and reclosing of channels. This implies that $\mathrm{Cx} 43$ is gating with positive and $\mathrm{Cx} 45$ with negative polarity. However, to rescue this interpretation, it has to be assumed that $\mathrm{HC}$ docking inverts the gating polarity of $\mathrm{Cx} 43$ (Desplantez et al., 2004). The smooth curves represent the best fit of data to the Boltzmann equation. The fitting parameters are also given in Table 2.

In conclusion, in each type of preparation, $g_{\mathrm{j}, \mathrm{ss}}=f\left(V_{\mathrm{j}}\right)$ shows a strong asymmetry. Conceivably, this indicates superimposed $V_{\mathrm{j}}$ gating; i.e., both $\mathrm{HCs}$ of a GJC may respond at a given $V_{\mathrm{j}}$. As a result, not all GJCs are open at $V_{\mathrm{j}}=0 \mathrm{mV}$; a small fraction is closed in the case of $\mathrm{Cx} 40$ $\mathrm{Cx} 43$ and a large fraction in the case of $\mathrm{Cx} 40-\mathrm{Cx} 45$. Hence, at rest $g_{\mathrm{j}, \mathrm{ss}}$ depends on both the number of channels and their functional state.

Data on single channels are rare. Cx40-Cx43 GJs have been most extensively studied (Valiunas et al., 2000; see also Cottrell \& Burt, 2001; Cottrell et al., 2002). Unitary currents are larger when cell $\mathrm{Cx} 43$ is negative inside and smaller when it is positive. In the latter case, residuals are virtually absent. The $\gamma_{\mathrm{j} \text {,main }}$ is $100 / 60 \mathrm{pS}$ at $V_{\mathrm{j}}= \pm 70 \mathrm{~m} V$. Interestingly, the channels exhibit a strong dependence on $V_{\mathrm{j}}$ polarity but a weak dependence on $V_{\mathrm{j}}$ amplitude. These properties are consistent with the behavior of $\mathrm{Cx} 40-\mathrm{Cx} 43$ at the multichannel level. In the case of $\mathrm{Cx} 40-\mathrm{Cx} 45$, the data are rudimentary. The only information available is a $\gamma_{\mathrm{j} \text {,main }}$ of 30-40 $\mathrm{pS}$ (V. Valiunas, unpublished). In the case of Cx43-Cx45, the situation is similar. These channels have a $\gamma_{\mathrm{j} \text {,main }}$ of $40 \mathrm{pS}$ and a $\gamma_{\mathrm{j}, \text { residual }}$ of $11 \mathrm{pS}$ (T. Desplantez \& R. Weingart, unpublished). Homomeric-heterotypic channels consist of two HCs of different conductance arranged in series. Hence, the conductance of a GJC can be predicted from the properties of both HCs: $\gamma_{\mathrm{j}, \text { main }}=\left(\gamma_{\mathrm{hc1}} \cdot \gamma_{\mathrm{hx} 2}\right) /$ $\left(\gamma_{\mathrm{hc} 1}+\gamma_{\mathrm{hc} 2}\right)$. The calculated values are 88,53 and $43 \mathrm{pS}$ for $\mathrm{Cx} 40-\mathrm{Cx} 43, \mathrm{Cx} 40-\mathrm{Cx} 45$ and $\mathrm{Cx} 43-\mathrm{Cx} 45$, respectively, and are in reasonable agreement with the experimental data. Likewise, a nonlinear function, $\gamma_{\text {hc,main }}=f\left(V_{\mathrm{m}}\right)$, results in nonlinearity for $\gamma_{\mathrm{j}, \text { main }}=f\left(V_{\mathrm{j}}\right)$.

The complex $I_{\mathrm{j}}$ records limit a quantitative kinetic analysis. However, in the case of $\mathrm{Cx} 40-\mathrm{Cx} 43, I_{\mathrm{j}}$ records and $g_{\mathrm{j}, \mathrm{ss}}=f\left(V_{\mathrm{j}}\right)$ indicate that channel gating almost exclusively occurs at negative $V_{\mathrm{j}}$, giving rise to a rapid $I_{\mathrm{j}}$ inactivation. This suggests superimposed gating; i.e., both gates of a GJC respond quasi-simultaneously. In the case of Cx40-Cx $45, I_{\mathrm{j}}$ records and $g_{\mathrm{j}, \mathrm{ss}}=f\left(V_{\mathrm{j}}\right)$ indicate that channel gating occurs at negative and positive $V_{\mathrm{j}}$, reflecting rapid inactivation and slow recovery from inactivation, respectively. This suggests sequential gating; i.e., one gate of a GJC responds after the other. In the case of $\mathrm{Cx} 43-\mathrm{Cx} 45, I_{\mathrm{j}}$ records and $g_{\mathrm{j}, \mathrm{ss}}=f\left(V_{\mathrm{j}}\right)$ indicate that channel gating mainly occurs at negative but also at positive $V_{\mathrm{j}}$, leading to rapid inactivation and slow recovery from inactivation, respectively. This suggests partial overlap of superimposed and sequential gating.

In summary, the properties of homomeric-heterotypic GJCs do not emerge readily from homomeric-homotypic GJCs. The former show properties not seen in the latter. Docking of HCs may influence the properties of GJCs being formed and thereby alter the Boltzmann parameters. Currently, analytic tools to describe and better understand the kinetic behavior of these channels are lacking.

\section{GJCs Made of Heteromeric Hemichannels}

Functional evidence for the existence of heteromeric GJCs made of $\mathrm{Cx} 40, \mathrm{Cx} 43$ and $\mathrm{Cx} 45$ is indirect (Elenes, Rubart \& Moreno, 1999; He et al., 1999; Cottrell \& Burt, 2001; Cottrell et al., 2002; Valiunas et al., 2001; Martinez et al., 2002; Polontchouk et al. 2002; Desplantez et al., 2004). The experimental approach has involved investigation of cell pairs with one cell expressing two different Cxs and the other cell expressing one type of $\mathrm{Cx}$ or the other or both. The strategy then was to identify channels with properties different from those already known, i.e., homomeric-homotypic and homomeric-heterotypic GJCs (see Fig. 7).

GJCs consisting of heteromeric $\mathrm{Cx} 40 / \mathrm{Cx} 43$ HCs have been identified biochemically and electrophysiologically (He et al., 1999; Cottrell \& Burt, 2001; Valiunas et al., 2001; Cottrell et al., 2002). At the multichannel level, the profiles of $g_{\mathrm{j}, \mathrm{ss}}=f\left(V_{\mathrm{j}}\right)$ can be nearly fitted with a combination of those of their homomeric $\mathrm{HC}$ counterparts. At the single-channel level, many conductances resemble those of the homomeric HC counterparts. This suggests that these channels follow the behavior of homotypic and heterotypic channels or that a sizable fraction of channels are nonfunctional. So far, no data are available on the formation and properties of channels made of heteromeric $\mathrm{Cx} 40 / \mathrm{Cx} 45$ HCs. With regard to $\mathrm{Cx} 43$ and $\mathrm{Cx} 45$, biochemical data suggest extensive formation of heteromeric HCs (Martinez et al., 2002). At the multichannel level, formation of GJCs made of heteromeric HCs is evident in $\mathrm{Cx} 43-\mathrm{Cx} 43 / 45$, Cx 45-Cx43/45 and Cx43/45-Cx43/45 cell pairs (Desplantez et al., 2004). Figure 7 illustrates results obtained from pairs in which the cells coexpress $\mathrm{Cx} 43$ and $\mathrm{Cx} 45$. The $I_{\mathrm{j}}$ signals vary from monotonic to nonmonotonic decays and transient increases (Fig. 7a). Each pair examined exhibits a unique $g_{\mathrm{j}, \mathrm{ss}}=f\left(V_{\mathrm{j}}\right)$ profile, suggesting the presence of $\mathrm{Cx} 43 / \mathrm{Cx} 45$ $\mathrm{Cx} 43, \mathrm{Cx} 43 / \mathrm{Cx} 45-\mathrm{Cx} 45$ and $\mathrm{Cx} 43 / \mathrm{Cx} 45-\mathrm{Cx} 43 / \mathrm{Cx} 45$ channels, besides $\mathrm{Cx} 43-\mathrm{Cx} 43, \mathrm{Cx} 45-\mathrm{Cx} 45$ and $\mathrm{Cx} 43-\mathrm{Cx} 45$ channels (Fig. 7b). Single-channel records provide evidence for conductances not seen in cells made of 
Fig. 7 Heteromeric-heterotypic GJs. Dependence of GJ conductance on junctional potential of cell pairs that coexpress $\mathrm{Cx} 43$ and $\mathrm{Cx} 45$. (A) Examples with a nearly symmetrical behavior. (B) Examples with a nonsymmetrical behavior. Upper panels: Superimposed records of GJ currents elicited by a standard pulse protocol. Lower panels: Normalized instantaneous $(\bigcirc)$ and steadystate $(\bullet)$ conductance as functions of $V_{\mathrm{j}}$. Source: (Desplantez et al., 2004)
A
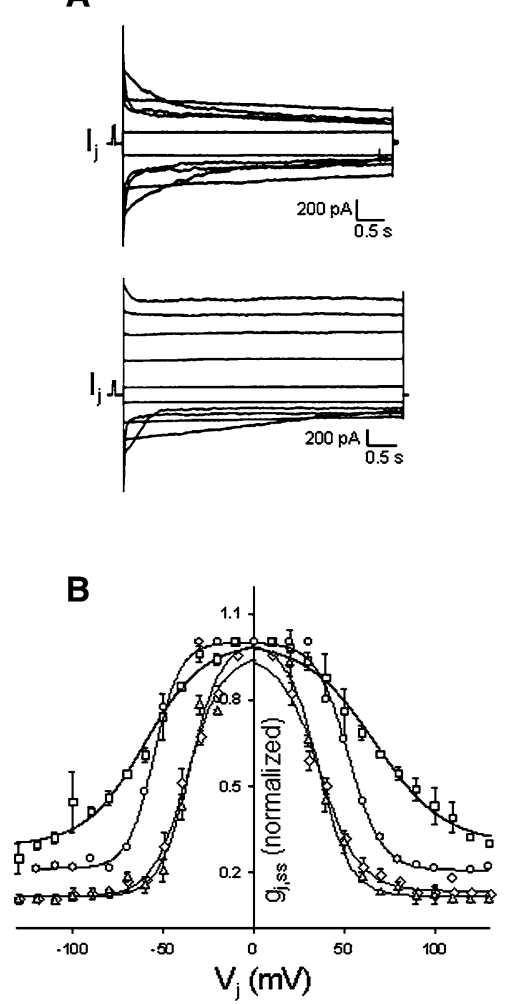

$C \times 43 / C \times 45-C x 43 / C \times 45$
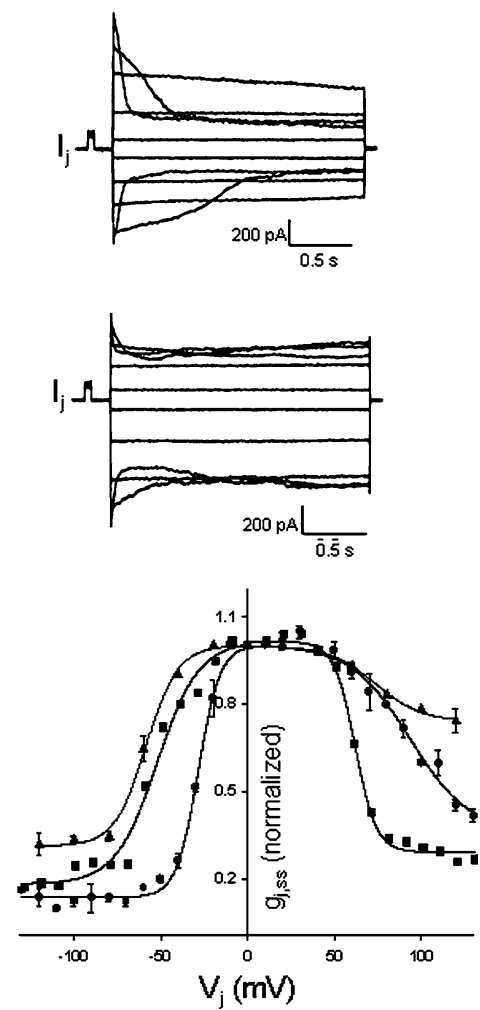

homomeric $\mathrm{HCs}$, suggesting extensive formation of heteromeric channels (Martinez et al., 2002).

Work in progress examines GJCs containing heteromeric HCs of known Cx composition (Desplantez et al., 2005; Weingart et al., 2006). The approach involves engineering $\mathrm{Cx}$ tandem constructs by linking directly, via the carboxy domain and the amino domain of $\mathrm{Cx} 1$ and $\mathrm{Cx} 2$, respectively, two cDNA sequences of two $\mathrm{Cx}$ isoforms, $\mathrm{Cx} 1 \sim \mathrm{Cx} 2$, in a circular plasmid. The STOP codon of $\mathrm{Cx} 1$ and the MET codon of $\mathrm{Cx} 2$ are replaced by a dipeptide, Leu-Glu. Nine constructs have been made: Cx $43 \sim \mathrm{Cx} 40, \quad \mathrm{Cx} 43 \sim \mathrm{Cx} 43, \quad \mathrm{Cx} 43 \sim \mathrm{Cx} 45, \quad \mathrm{Cx} 45 \sim \mathrm{Cx} 43$, $\mathrm{Cx} 45 \sim \mathrm{Cx} 40, \mathrm{Cx} 45 \sim \mathrm{Cx} 45, \mathrm{Cx} 40 \sim \mathrm{Cx} 40, \mathrm{Cx} 40 \sim \mathrm{Cx} 43$ and $\mathrm{Cx} 40 \sim \mathrm{Cx} 45$. These have been transfected into HeLa cells, and stable clones have been obtained for each. Immunoblots and immunohistochemistry reveal that all fusion proteins are properly translated and inserted at cell-cell contact sites (see Fig. 8). Preliminary electrophysiologicaly experiments reveal that all constructs form functional GJCs. Electrical recordings performed on cell pairs for each of these tandems are currently producing novel data (reports in preparation).

\section{Effects of GJCs on Impulse Propagation}

As outlined above (see Propagation of the Cardiac Impulse), conduction velocity is primarily determined by the excit- atory inward current (i.e., $I_{\mathrm{Na}}, I_{\mathrm{Ca}}$ ) as a source factor and GJCs as sink factors. With regard to GJCs, the following parameters are critically involved: (1) channel number, (2) channel conductance, (3) voltage gating of channels, (4) channel kinetics and (5) channel orientation (in the case of mixed channels). These parameters provide the framework for a discussion on GJCs and impulse propagation.

Homomeric-Homotypic Channels

\section{Static aspects}

The functional state of GJs at rest is given by the function $g_{\mathrm{j}, \text { inst }}=f\left(V_{\mathrm{j}}\right)$. It is determined by the number of operational channels, $N$, the unitary conductance, $\gamma_{\mathrm{j}, \text { main }}$, and the $V_{\mathrm{j}}$ sensitivity of $\gamma_{\mathrm{j} \text {,main }}$. At $V_{\mathrm{j}}=0 \mathrm{~m} V, g_{\mathrm{j}, \text { inst }}=N \cdot \gamma_{\mathrm{j} \text {,main }}$, provided $\gamma_{\mathrm{j} \text {,main }}$ is extrapolated to $V_{\mathrm{j}}=0 \mathrm{mV}$. Since $g_{\mathrm{j} \text {,inst }}$ and $g_{\mathrm{j}, \mathrm{ss}}$ are maximal at $V_{\mathrm{j}}=0 \mathrm{mV}$, all channels must be open. Hence, $g_{\mathrm{j} \text {,inst }}$ at $V_{\mathrm{j}}=0 \mathrm{mV}$ is a convenient measure for the basic component of the static aspects of GJs. The larger $N$ and $\gamma_{\mathrm{j}, \text { main }}$, the faster is the conduction velocity, $\theta$. Channels made of $\mathrm{Cx} 40, \mathrm{Cx} 43$ and $\mathrm{Cx} 45$ have a $\gamma_{\mathrm{j} \text {,main }}$ of 162, 61 and $32 \mathrm{pS}$, respectively (see Table 2). Hence, the propensity to contribute to $\theta$ obeys the sequence $\mathrm{Cx} 40>$ $\mathrm{Cx} 43>\mathrm{Cx} 45$. The function $g_{\mathrm{j}, \text { inst }}=f\left(V_{\mathrm{j}}\right)$ exhibits a smooth curve. Its curvature depends on the type of $\mathrm{Cx}$. Hence, it is a measure for the modifying component of the static 

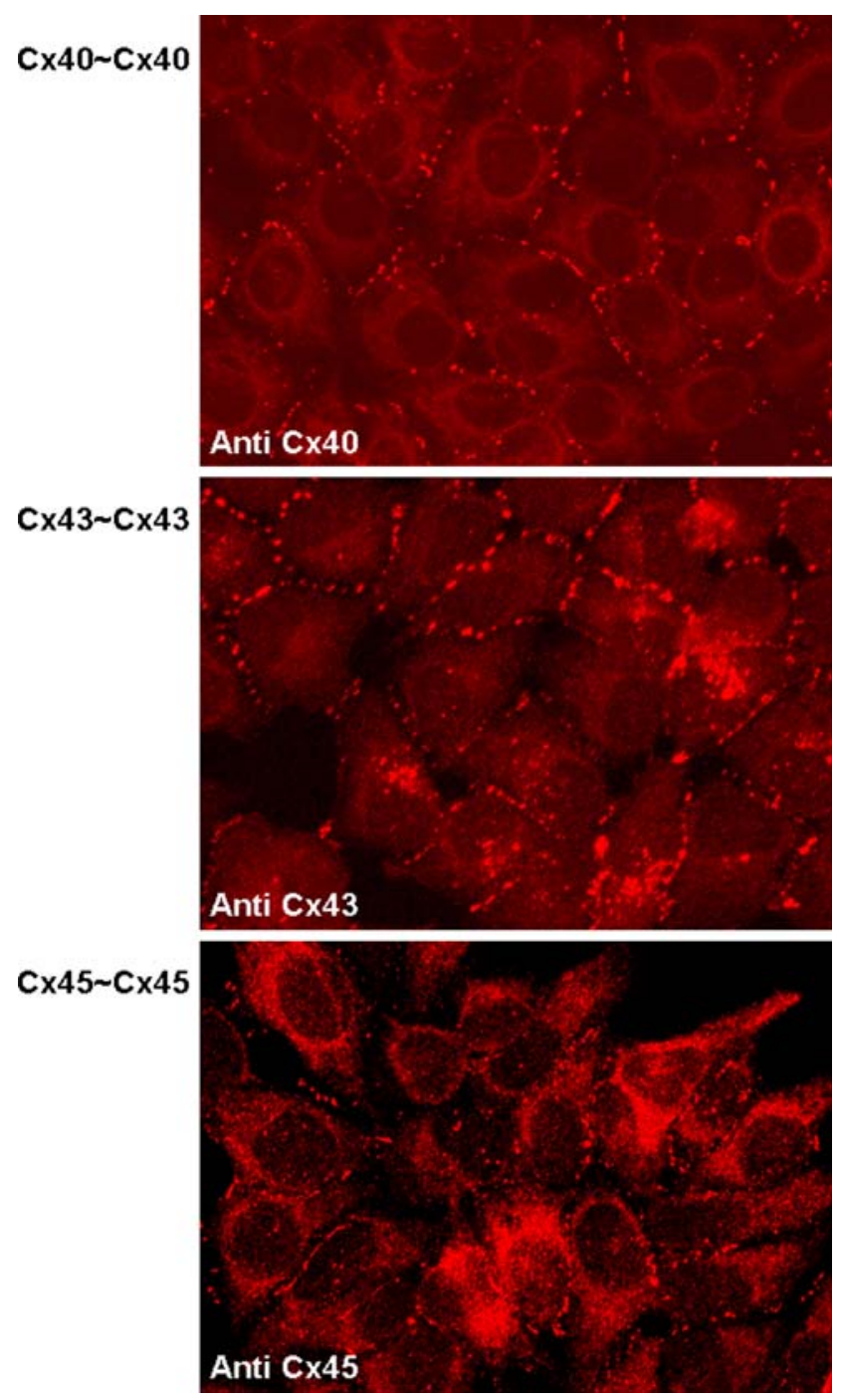

Fig. 8 Immunostaining of HeLa cells transfected with the twintandem constructs. Specific antibodies against $\mathrm{Cx} 40, \mathrm{Cx} 43$ and $\mathrm{Cx} 45$ clearly label cell interfaces in the punctate pattern typical of GJs. This indicates proper folding, connexon oligomerization and docking in the extracellular space

aspects of GJs. The stronger the curvature, the larger is the effect on $\theta$. Overall, the $V_{\mathrm{j}}$ sensitivity of $g_{\mathrm{j} \text {,inst }}$ slightly reduces $\theta$, most notably for $\mathrm{Cx} 45-\mathrm{Cx} 45$.

\section{Dynamic aspects}

The functional state of GJs alters during impulse propagation and thereby affects $\theta$. This is because $g_{\mathrm{j}, \text { inst }}=f\left(V_{\mathrm{j}}\right)$ gives way to the bell-shaped function $g_{\mathrm{j}, \mathrm{ss}}=f\left(V_{\mathrm{j}}\right)$ (see Fig. 5). Two properties can interfere with $\theta$, the voltage sensitivity of $g_{\mathrm{j}, \mathrm{ss}}$ and the kinetics of $g_{\mathrm{j}, \mathrm{ss}}$. With regard to $V_{\mathrm{j}}$ sensitivity, $\mathrm{Cx} 45$ is most sensitive and $\mathrm{Cx} 43$ least sensitive, as evidenced by the $V_{\mathrm{j}, 0}$ and $z$ data (see Table 2). At a given $V_{\mathrm{j}}$, the decrease of $g_{\mathrm{j}, \mathrm{ss}}$ is largest for $\mathrm{Cx} 45$ and smallest for $\mathrm{Cx} 43$; i.e., $\mathrm{Cx} 45$ slows down $\theta$ most prominently and $\mathrm{Cx} 43$ least prominently. With regard to kinetics, $I_{\mathrm{j}}$ inactivation is equally fast for $\mathrm{Cx} 40$ and $\mathrm{Cx} 43$ and slightly slower for $\mathrm{Cx} 45$. The faster the inactivation, the larger is the reduction of $\theta$. Hence, $\mathrm{Cx} 40$ and Cx43 impair $\theta$ slightly more than Cx45. Taken together, the reduction of $\theta$ by the dynamic aspects follows the sequence $\mathrm{Cx} 45>\mathrm{Cx} 40=\mathrm{Cx} 43$. The recovery of $I_{\mathrm{j}}$ from inactivation plays no role at normal conditions (heart frequency, 40-80 beats/min; action potential duration, $200-300 \mathrm{~ms}$ ). However, it can become relevant due to incomplete recovery of $I_{\mathrm{j}}$ at short diastole (e.g., 150 beats/min, $150-250 \mathrm{~ms}$ ), for instance during physical stress.

\section{Prominent tissues}

Ventricular muscle contains $\mathrm{Cx} 43$. The expression level is high and the channels have a medium $\gamma_{\mathrm{j} \text {,main }}$ (see Tables 1 and 2). Hence, the basic static aspects $\left(N, \gamma_{\mathrm{j}, \text { main }}\right)$ are consistent with the rapid conduction velocity $(\theta \cong 0.75 \mathrm{~m} / \mathrm{s}$, see Table 3) and safe impulse propagation reported. The impairment by the modifying component of the static aspects and by the dynamic aspects are small and medium, respectively; hence, the overall effect on $\theta$ is little to moderate.

SA and AV nodes contain Cx45. The expression level is moderate in both tissues and the channels have a low $\gamma_{\mathrm{j} \text {,main }}$ (see Tables 1 and 2). Hence, the basic static aspects tend toward a slow conduction velocity $(\theta=0.03-0.05 \mathrm{~m} / \mathrm{s}$, see Table 3 ). The low cell connectivity may also contribute. The impairment by the modifying component of the static aspects and the dynamic aspects are medium and large, respectively. Overall, they contribute noticeably to the low $\theta$.

Atrial muscle contains $\mathrm{Cx} 40$ and $\mathrm{Cx} 43$. The expression level is equally high for both and comparable to that of Cx43 in ventricular muscle (see Table 1). This allows the formation of $\mathrm{Cx} 40-\mathrm{Cx} 40$ and $\mathrm{Cx} 43-\mathrm{Cx} 43$ channels, although in small number (see below). $\mathrm{Cx} 40-\mathrm{Cx} 40$ channels have a large $\gamma_{\mathrm{j} \text {,main }}$ (see Table 2), predicted to give a large $\theta$. The impairment by the modifying component of the static aspects and the dynamic aspects is small and medium, respectively. Hence, the overall effect on $\theta$ is little to moderate. The role of $\mathrm{Cx} 43-\mathrm{Cx} 43$ channels in atrial muscle is identical to that in ventricular muscle (see above). Taken together, based on the basic static aspects, coexistence of $\mathrm{Cx} 40-\mathrm{Cx} 40$ and $\mathrm{Cx} 43-\mathrm{Cx} 43$ channels predicts a $\theta$ faster than that of ventricular muscle. Yet, the value observed is considerably slower $(\theta=0.6 \mathrm{~m} / \mathrm{s}$, see Table 3). This likely indicates the existence of a large population of mixed channels of lower $\gamma_{j \text {,main }}$ and/or nonfunctional channels (see below). The combined impairment by the static aspects and by the dynamic aspects is small and medium, respectively; hence, the effect on $\theta$ is little to moderate. 
Table 3 Parameters determining the effective voltage across a GJ during a propagated action potential

\begin{tabular}{|c|c|c|c|c|c|c|c|}
\hline Cell type & $l(\mu \mathrm{m})$ & $\lambda(\mathrm{mm})$ & $\theta(\mathrm{m} / \mathrm{s})$ & $A_{\mathrm{AP}}(\mathrm{m} V)$ & $t_{\text {upstroke }}(\mathrm{ms})$ & $l_{\text {upstroke }}(\mathrm{mm})$ & $\Delta V / n(\mathrm{~m} V)$ \\
\hline SA node & 50 & 0.47 & 0.03 & 60 & 25 & 2.5 & 1.2 \\
\hline Auricle & 100 & 0.66 & 0.6 & 110 & 2 & 0.7 & 18.3 \\
\hline AV node & 50 & - & 0.05 & 60 & 25 & 2.5 & 1.2 \\
\hline Purkinje fiber & 150 & 1.9 & 2.3 & 125 & 0.5 & 1.25 & 18 \\
\hline Ventricle & 100 & 0.88 & 0.75 & 100 & 2 & 0.7 & 17 \\
\hline
\end{tabular}

$l$, cell length; $\theta$, conduction velocity; $A_{\mathrm{AP}}$, amplitude of action potential; $t_{\text {upstroke }}$, duration of upstroke; $l_{\text {upstroke }}$, length of upstroke; $\Delta V$, voltage gradient; $n$, number of GJs. Sources: $\lambda, \theta$ (Kléber, Janse \& Fast, 2001); other parameters from various monographs or calculated

Purkinje fibers, like atrial muscle, express $\mathrm{Cx} 40$ and Cx43 at high levels and can form three types of channels (see above) besides mixed channels (see below). They show a value of $\theta$ three to four times faster than that of atrial or ventricular muscle $(\theta=2.3 \mathrm{~m} / \mathrm{s}$, see Table 3$)$. This is consistent with the observation that $\mathrm{Cx} 43$ is preferentially localized in the distal part of the tissue (see above, $\mathrm{Cx}$ Expression in the Normal Adult Heart).

\section{Homomeric-Heterotypic Channels}

\section{Static aspects}

The functional state of GJs at rest is still given by the relationship between $g_{\mathrm{j} \text {,inst }}$ and $V_{\mathrm{j}}$. However, $g_{\mathrm{j}, \mathrm{ss}}$ may not be maximal at $V_{\mathrm{j}}=0 \mathrm{mV}$; i.e., some channels are in the main state and others in the residual state. Therefore, $g_{\mathrm{j}, \mathrm{ss}}=\left(m \cdot \gamma_{\mathrm{j}, \text { main }}\right)+\left(r \cdot \gamma_{\mathrm{j}, \text { residual }}\right)$, where $N=m+r(m, r$, number of channels in the main and residual states). Hence, the expression level of Cxs together with $\gamma_{\mathrm{j}, \text { main }}$ may no longer be useful to make predictions of $\theta$. While $g_{\mathrm{j}, \mathrm{ss}}=f\left(V_{\mathrm{j}}\right)$ still has a maximum around $V_{\mathrm{j}}=0 \mathrm{~m} V$ in the case of $\mathrm{Cx} 40-\mathrm{Cx} 43$, this is no longer true for $\mathrm{Cx} 40-\mathrm{C} 45$ and $\mathrm{Cx} 43-\mathrm{Cx} 45$. In these cases, the maximum is shifted to a negative and positive $V_{\mathrm{j}}$, respectively. Moreover, $g_{\mathrm{j}, \text { inst }}=f\left(V_{\mathrm{j}}\right)$ is no longer symmetrical. Its asymmetry documents an instantaneous rectification. Hence, $\theta$ now also depends on channel orientation. Asymmetry of $g_{\mathrm{j} \text {,inst }}$ exists for $\mathrm{Cx} 40-\mathrm{Cx} 43, \mathrm{Cx} 40-\mathrm{Cx} 45$ and $\mathrm{Cx} 43-\mathrm{Cx} 45$ channels, most prominently for $\mathrm{Cx} 40-\mathrm{Cx} 43$. Hence, when considering the basic static aspects of GJs, these facts should be kept in mind.

\section{Dynamic aspects}

The asymmetry of $g_{\mathrm{j}, \text { inst }}=f\left(V_{\mathrm{j}}\right)$ has its equivalent in the function $g_{\mathrm{j}, \mathrm{ss}}=f\left(V_{\mathrm{j}}\right)$, introducing a steady-state rectification. This is because $V_{\mathrm{j}}$ sensitivity and kinetics of gating are no more symmetrical with regard to $V_{\mathrm{j}}=0 \mathrm{~m} V$; instead, both depend on $V_{\mathrm{j}}$ polarity. Hence, the dynamic aspects now include $V_{\mathrm{j}}$ sensitivity of $g_{\mathrm{jss}}$, kinetics of $g_{\mathrm{j}}$ and gating direction, i.e., inactivation $v s$. recovery from inactivation. A combination of these criteria leads to the following response patterns. $\mathrm{Cx} 40-\mathrm{Cx} 43$ inactivates at fast speed and with medium $V_{\mathrm{j}}$ sensitivity when cell $\mathrm{Cx} 40$ is positive inside and shows virtually no sign of gating when cell $\mathrm{Cx} 40$ is negative inside. $\mathrm{Cx} 40-\mathrm{Cx} 45$ recovers from inactivation at slow speed and with medium $V_{\mathrm{j}}$ sensitivity when cell $\mathrm{Cx} 40$ is negative inside and inactivates at fast speed and with high $V_{\mathrm{j}}$ sensitivity when cell $\mathrm{Cx} 40$ is positive inside. $\mathrm{Cx} 43$ Cx45 inactivates at medium speed and with high $V_{\mathrm{j}}$ sensitivity when cell $\mathrm{Cx} 43$ is positive inside and recovers from inactivation at slow speed and with low $V_{\mathrm{j}}$ sensitivity when cell $\mathrm{Cx} 43$ is negative inside.

\section{Prominent tissues}

Atrial muscle, bundle branch and Purkinje fibers preferentially express $\mathrm{Cx}_{4} 40$ and $\mathrm{Cx} 43$. The expression level for both Cxs is high to medium. Besides few $\mathrm{Cx} 40-\mathrm{Cx} 40$ and $\mathrm{Cx} 43-\mathrm{Cx} 43$ channels (see above), these tissues may in theory also form some $\mathrm{Cx} 40-\mathrm{Cx} 43$ channels exhibiting a large $\gamma_{\mathrm{j} \text {,main }}$ (see Table 2). The basic static aspects predict a fast $\theta$ for these channels. This conclusion seems appropriate since $g_{\mathrm{j}, \mathrm{ss}}=f\left(V_{\mathrm{j}}\right)$ suggests that all channels are open at $V_{\mathrm{j}}=0 \mathrm{~m} V$. However, while it is consistent with experimental data for bundle branch and Purkinje fibers (notably in the distal zone), it is not for atrial muscle. A likely explanation is that the latter mainly expresses heteromeric channels of low $\gamma_{\mathrm{j} \text {,main }}$ (see below). As to $\mathrm{Cx} 40-\mathrm{Cx} 43$ channels, with regard to modifying component of the static aspects, $g_{\mathrm{j} \text {,inst }}=f\left(V_{\mathrm{j}}\right)$ has a negative slope. Hence, impulse propagation is initially moderately facilitated from $\mathrm{Cx} 40$ to $\mathrm{Cx} 43$ and impaired from $\mathrm{Cx} 43$ to $\mathrm{Cx} 40$. With regard to the dynamic aspects, $V_{\mathrm{j}}$ sensitivity of $g_{\mathrm{j}, \mathrm{ss}}$ and kinetics of $g_{\mathrm{j}}$ counteract the facilitation with time at negative $V_{\mathrm{j}}$ but maintain the impairment at positive $V_{\mathrm{j}}$. As a result, initially impulse propagation from $\mathrm{Cx} 40$ to $\mathrm{Cx} 43$ is transiently facilitated. If there were random orientation of $\mathrm{Cx} 40-\mathrm{Cx} 43$ GJCs in the cells within the tissue, overall the facilitating effect would be annulled. Alternatively, inhomogeneous distribution of $\mathrm{Cx} 40-\mathrm{Cx} 43$ GJCs could potentially lead to 
structural compartmentalization and, hence, to functional compartmentalization.

Purkinje fibers express Cx40 and Cx43 (preferentially in the distal zone), and ventricular muscle expresses Cx43 at a high level (see Table 1). Besides Cx40-Cx40, this offers the theoretical possibility of formation of $\mathrm{Cx} 40-\mathrm{Cx} 43$ channels in the distal zone of Purkinje fibers and in the transitional zone between Purkinje fibers and ventricular muscle. Since Cx40-Cx43 channels would initially facilitate impulse propagation from $\mathrm{Cx} 40$ to $\mathrm{Cx} 43$, orthodromic propagation would be transiently and moderately facilitated early during action potentials. If anything, coexistence of $\mathrm{Cx} 43-\mathrm{Cx} 43$ and $\mathrm{Cx} 40-\mathrm{Cx} 40$ channels tends to dampen the properties of any Cx40-Cx43 channels. Similar structural and functional conditions prevail at His bundlebundle branch and bundle branch-Purkinje fiber tissue junctions (see below). In both cases, orthodromic impulse propagation is transiently and moderately facilitated early during action potentials.

SA node expresses $\mathrm{Cx} 45$ at medium level, and atrial muscle expresses $\mathrm{Cx} 40$ and $\mathrm{Cx} 43$ at high level (see Table 2). This offers the possibility to form $\mathrm{Cx} 40-\mathrm{Cx} 45$ and Cx43-Cx45 channels in the transitional zone between these tissues. Cx40-Cx45 channels have a medium $\gamma_{\mathrm{j} \text {,main }}$ (see Table 2). With regard to the basic static aspects, $g_{\mathrm{j}, \mathrm{ss}}=f\left(V_{\mathrm{j}}\right)$ indicates that $g_{\mathrm{j}, \mathrm{ss}}$ at $V_{\mathrm{j}}=0 \mathrm{mV}$ is far from maximal; i.e., about $50 \%$ of the channels are in the residual state. Hence, $\theta$ is accordingly slower at resting conditions. With regard to the modifying component of the static aspects, $g_{\mathrm{j} \text {,inst }}=f\left(V_{\mathrm{j}}\right)$ has a positive slope at negative $V_{\mathrm{j}}$ and vice versa. Hence, initially impulse propagation is slightly impaired at both $V_{\mathrm{j}}$ polarities. With regard to the dynamic aspects, channel opening leads to a prominent maintained facilitation from $\mathrm{Cx} 45$ to $\mathrm{Cx} 40$ that develops gradually. As a result, orthodromic propagation is slightly impaired early during action potentials, with strong and ongoing facilitation later on.

Cx43-Cx45 channels also have a medium $\gamma_{\mathrm{j} \text {,main }}$ (see Table 2). As to the basic static aspects, $g_{\mathrm{j}, \mathrm{ss}}=f\left(V_{\mathrm{j}}\right)$ indicates that $g_{\mathrm{j}, \mathrm{ss}}$ at $V_{\mathrm{j}}=0 \mathrm{mV}$ is less than submaximal; i.e., about $75 \%$ of the channels are in the residual state. Therefore, $\theta$ is accordingly slower at resting conditions. As to the modifying component of the static aspects, $g_{\mathrm{j} \text {,inst }}=f\left(V_{\mathrm{j}}\right)$ has a positive slope at negative $V_{\mathrm{j}}$ and remains constant at positive $V_{\mathrm{j}}$. Hence, initially impulse propagation undergoes no change at positive $V_{\mathrm{j}}$. As to the dynamic aspects, channel opening provokes a prominent facilitation from $\mathrm{Cx} 45$ to $\mathrm{Cx} 40$. The change occurs gradually and is maintained. As a result, orthodromic propagation is unaffected early during action potentials and substantially facilitated in a sustained manner later. Taken together, the facilitation by both $\mathrm{Cx} 40-\mathrm{Cx} 45$ and $\mathrm{Cx} 43-\mathrm{Cx} 45$ channels is orthodromic.
The tissue junctions AV node-His bundle and His bundle-bundle branch resemble the junction SA node-atrial muscle with regard to expression and distribution of $\mathrm{Cx} 45$ (leading tissue) and $\mathrm{Cx} 40$ and $\mathrm{Cx} 43$ (follower tissue). Hence, they also resemble each other functionally. This means their $\mathrm{Cx} 40-\mathrm{Cx} 45$ and $\mathrm{Cx} 43-\mathrm{Cx} 45$ channels facilitate impulse propagation from the leading to the follower tissue, i.e., orthodromically.

His bundle expresses $\mathrm{Cx} 40$ and $\mathrm{Cx} 45$ at medium level and $\mathrm{Cx} 43$ at low level (see Table 1). Hence, it is the most versatile tissue with regard to channel formation. Besides three kinds of homotypic channels and three kinds of heterotypic channels (see above), it is likely to contain heteromeric channels, presumably in large numbers (see below). Assuming random orientation of the heterotypic channels within the tissue, their facilitating and impairing effects would be annulled. Alternatively, compartmentalized channel expression may lead to functional compartmentalization.

\section{GJCs Made of Heteromeric HCs}

Quantitative data on GJCs containing heteromeric HCs of known Cx composition is rare; hence, discussion of the influence on $\theta$ is difficult. Preliminary studies of cells expressing $\mathrm{Cx}$ tandems indicate that these channels operate. However, beyond this we can only speculate. While some properties of homomeric-heterotypic channels can be predicted from simpler channels, this is not possible for heteromeric channels. Assuming that there are no interactions between the $\mathrm{Cxs}$ of an $\mathrm{HC}$, the expectations are that the properties of heteromeric GJCs range from those of homomeric-homotypic channels to those of homomericheterotypic channels.

Given that heteromeric GJCs exist, the question arises as to how many structurally different GJCs with potentially different functional properties are possible. Assuming two different $\mathrm{Cxs}$ at equal amounts and random oligomerization of Cxs, this leads to a binomial distribution with 14 different $\mathrm{HCs}$, i.e., two homomerics and 12 heteromerics. Likewise, assuming random docking between these HCs, this results in a binomial distribution with 196 different GJCs, i.e., two homomeric-homotypic, one homomericheterotypic, 12 heteromeric-homotypic and 181 heteromeric-heterotypic channels. Allowing rotational symmetry of HCs, we would end up with $6 \cdot 196=1,176$ GJCs, of which 28 are nonheteromerics, i.e., 2.5\%. Hence, the incidence of these last is by far the largest. Even more structurally and functionally different channels would result in the case of three different Cxs. Obviously, unequal $\mathrm{Cx}$ expression and/or nonrandom assembly processes would greatly affect the frequency of the different types of channels. 


\section{Prominent tissues}

His bundle contains $\mathrm{Cx} 40, \mathrm{Cx} 45$ and $\mathrm{Cx} 43$ (in some species) expressed at medium, medium and small levels, respectively. Hence, it is predestined to form a large number of different heteromeric channels. Atrial muscle, bundle branch and Purkinje fibers express all three Cxs at different levels and, hence, belong to this group of tissues as well. Transitional zones prone to form heteromeric channels include the junctions between all tissues of the heart, i.e., SA node-atrial muscle, atrial muscle-AV node, AV node-His bundle, His bundle-bundle branch, bundle branch-Purkinje fiber and Purkinje fiber-ventricular muscle.

Atrial muscle is of particular interest and may serve as model for coexpressing tissue. It expresses $\mathrm{Cx} 40$ and $\mathrm{Cx} 43$ at equal levels, as high as that of $\mathrm{Cx} 43$ in ventricular muscle. Based on this, it is expected to have a $\theta$ larger than that of ventricular muscle, yet the opposite is the case. This discrepancy hints at a strong contribution of heteromeric GJCs. Following the consideration that most channels are heteromeric (see above), the consequences would be that these channels exhibit a $\gamma_{\mathrm{j} \text {,main }}$ lower than that of $\mathrm{Cx} 40$ $\mathrm{Cx} 40$ or $\mathrm{Cx} 43-\mathrm{Cx} 43$ channels and/or not all heteromeric HCs form operational GJCs.

Only little is known about preferences to form particular HCs and GJCs. The case best examined is $\mathrm{Cx} 40 / \mathrm{Cx} 43$. Combined biochemical and electrophysiological data gained from transfected HeLa cells indicate that the coupling conductance in $\mathrm{Cx} 40-\mathrm{Cx} 43$ is reduced by $\sim 75 \%$ when compared with parental cell pairs (Valiunas et al., 2001). This indicates lowering of the docking efficiency, suggesting that some heteromeric HCs of certain stoichiometries are unable to establish functional channels because of structural incompatibilities. Thus, there may be a bias in the types of channels formed. The binomial distribution therefore cannot be used to predict accurately the percentage of heteromers, though they would still form the large majority.

Another study examined the regulatory interactions between $\mathrm{Cx} 40$ and $\mathrm{Cx} 43$ in neonatal atrial myocytes (Beauchamp et al., 2006). Myocytes of $\mathrm{Cx} 40$ and $\mathrm{Cx} 43$ knockout mice grown in synthetic strands were used to measure $\mathrm{Cx}$ expression and $\theta$. It turned out that reduced $\mathrm{Cx} 40$ expression was associated with an increase in $\theta$ instead of a decrease. In contrast, a decrease in $\mathrm{Cx} 43$ expression is accompanied by a decrease in $\theta$. This phenomenon is not seen in vivo. These data are consistent with the formation of heteromeric $\mathrm{Cx} 40 / \mathrm{Cx} 43$ channels. Presumably, this is under the control of a developmental gene-transcription program and may play a role in electrical remodeling during mechanical overload and hypertrophy.
Role of the Junctional Voltage Gradient

What is the significance of the dynamic biophysical properties of GJCs on impulse propagation in the heart? To answer this question, we have to know the voltage gradient experienced by a GJ during impulse propagation. Approximate values may be obtained as follows. The propagated action potential can be regarded as a depolarizing wave that propagates over the entire heart. Depending on the area, $\theta$ is as slow as $0.03 \mathrm{~m} / \mathrm{s}$ or as fast as $2.3 \mathrm{~m} / \mathrm{s}$ (see Table 3). The upstroke of the action potential experiences a maximal change in membrane potential (amplitude of action potential, $A_{\mathrm{AP}}$ ) within a few milliseconds (duration of upstroke, $t_{\text {upstroke }}$ ). From the values of $t_{\text {upstroke }}$ and $\theta$, the length over which the voltage change occurs can be calculated (length of upstroke, $l_{\text {upstroke }}$ ). Considering $l_{\text {upstroke }}$ and $A_{\mathrm{AP}}$, the overall voltage gradient along the tissue fiber axis can be estimated (voltage gradient, $\Delta V$ ). From $l_{\text {upstroke }}$ and the cell length, $l_{\text {cell }}$, the number of GJs (n) sensing the voltage gradient can be estimated as $n=\left(l_{\text {upstroke }} / l_{\text {cell }}\right)-1$. Table 3 summarizes the values of $\Delta V / n$ determined for the different cardiac tissues as well as the parameters used for the calculation. $\Delta V / n$ varies eightfold. The larger $\Delta V / n$, the more important is the modulatory role - exerted by the dynamic properties of GJCs - on $\theta$ during an action potential. The smaller $\Delta V / n$, the less important is the role. Hence, under normal conditions, Purkinje fibers, ventricular muscle and atrial muscle are expected to be most affected and SA node and AV node least affected.

In the pathophysiological setting, several reasons can give rise to slow impulse propagation. For example, during anoxia and ischemia, lowering of $\mathrm{pH}_{i}$ or $\mathrm{pCa}_{i}$ and accumulation of lipophilic agents (e.g., arachidonic acid) - via chemical gating (reviewed by Harris, 2001) - leads to a decrease in $g_{\mathrm{j}}$, thus giving rise to a decrease in $\theta$. In turn, this increases $\Delta V / n$ and hence further decreases $\theta$ via the modifying component of the static aspects and the dynamic aspects of GJCs (see above). In general, lowering of $\theta$ in cardiac diseases may exert the same effect.

\section{Concluding Remarks}

For many years, when discussing impulse propagation, GJs were regarded as static structures behaving as ohmic resistors. However, work over the last two decades has revealed that they are voltage-gated (reviewed by Harris, 2001). This property renders GJCs dynamic structures, able to alter the functional state during impulse propagation.

The goal of this review has been to examine impulse propagation in the heart in light of recent findings on the biophysical properties of GJCs in relation to the data on 
distribution and expression of the three major cardiac Cxs, i.e., $\mathrm{Cx} 43, \mathrm{Cx} 40$ and $\mathrm{Cx} 45$. The data presented on electrophysiological properties of homomeric-homotypic and homomeric-heterotypic GJCs are accurate, permitting predictions of $\theta$. However, with respect to heteromeric channels, such data are still lacking. As a working hypothesis, it may not be unrealistic to predict that the properties of heteromeric channels dwell between those of parental homomeric-homotypic and homomeric-heterotypic channels. Assuming equal $\mathrm{Cx}$ concentrations and random mixing, the contribution of the homomeric-homotypic and homomeric-heterotypic channels is expected to be small, i.e., in the low percentage range, the remainder being heteromeric channels.

The precise data on expression/localization of Cxs that are now available allow differences among $\mathrm{Cx}$ orthologues in cardiac tissues to be expressed quasi-quantitatively. As highlighted at the outset, the data in Table 1, on which the electrophysiological predictions are made, refer to our current understanding of the situation in the adult human heart. The Cx expression data on atria and ventricles are most robust, being derived from quantitative Western and Northern blots as well as immunocytochemistry. Those on the tissues of the conduction system, of necessity, rely on immunocytochemistry alone. It should finally be borne in mind that species-related differences in $\mathrm{Cx}$ expression patterns, both within the conduction system and elsewhere, would influence the specific electrophysiological predictions discussed.

Acknowledgements This review is dedicated to Prof. Werner Loewenstein on the occasion of his eightieth birthday. Supported by Swiss National Science Foundation (SNSF) (grant 3100A0-108175 to R. W.) and the British Heart Foundation (grant PG/02/083 to N. S. and E. D.).

\section{References}

Barrio LC, Capel J, Jarillo JA, Castro C, Revilla A (1997) Speciesspecific voltage-gating properties of connexin-45 junctions expressed in Xenopus oocytes. Biophys J 73:757-769

Beauchamp P, Yamada KA, Baertschi AJ, Green K, Kanter EM, Saffitz JE, Kleber AG (2006) Relative contributions of connexins 40 and 43 to atrial impulse propagation in synthetic strands of neonatal and fetal murine cardiomyocytes. Circ Res 99:12161224

Bukauskas FF, Elfgang C, Willecke K, Weingart R (1995) Biophysical properties of gap junction channels formed by mouse connexin40 in induced pairs of transfected human HeLa cells. Biophys J 68:2289-2298

Coppen SR, Kodama I, Boyett MR, Dobrzynski H, Takagishi Y, Honjo H, Yeh HI, Severs NJ (1999a) Connexin45, a major connexin of the rabbit sinoatrial node, is coexpressed with connexin 43 in a restricted zone at the nodal-crista terminalis border. J Histochem Cytochem 47:907-918

Coppen SR, Severs NJ (2002) Diversity of connexin expression patterns in the atrioventricular node: vestigial consequence or functional specialization? J Cardiovasc Electrophysiol 13:625626

Coppen SR, Severs NJ, Gourdie RG (1999b) Connexin45 (a6) expression delineates an extended conduction system in the embryonic and mature rodent heart. Dev Genet 24:82-90

Cottrell GT, Burt JM (2001) Heterotypic gap junction channel formation between heteromeric and homomeric $\mathrm{Cx} 40$ and $\mathrm{Cx} 43$ connexons. Am J Physiol 281:C1559-C1567

Cottrell TG, Wu Y, Burt JM (2002) Cx40 and Cx43 expression ratio influences heteromeric/heterotypic gap junction channel properties. Am J Physiol 282:C1469-C1482

Desplantez T, Halliday D, Dupont E, Weingart R (2004) Cardiac connexins $\mathrm{Cx} 43$ and $\mathrm{Cx} 45$ : formation of diverse gap junction channels with diverse electrical properties. Pfluegers Arch 448:363-375

Desplantez T, Dupont E, Thomas N, Severs NJ, Weingart R (2005) Tandem constructs of $\mathrm{Cx} 40, \mathrm{Cx} 43$ and $\mathrm{Cx} 45$ to probe properties of heteromeric channels of known composition. Abstracts of the International Gap Junction Conference, August 13-18, 2005, Whistler, BC, Canada, p 121

Dupont E, Matsushita T, Kaba R, Vozzi C, Coppen SR, Khan N, Kaprielian R, Yacoub MH, Severs NJ (2001) Altered connexin expression in human congestive heart failure. J Mol Cell Cardiol 33:359-371

Elenes S, Rubart M, Moreno AP (1999) Junctional communication between isolated pairs of canine atrial cells is mediated by homogeneous and heterogeneous gap junction channels. J Cardiovasc Electrophysiol 10:990-1004

Elenes S, Martinez AD, Delmar M, Beyer E, Moreno AP (2001) Heterotypic docking of $\mathrm{Cx} 43$ and $\mathrm{Cx} 45$ connexons blocks fast voltage gating of Cx43. Biophys J 81:1406-1418

Gourdie RG, Green CR, Severs NJ (1991) Gap junction distribution in adult mammalian myocardium revealed by an antipeptide antibody and laser scanning confocal microscopy. J Cell Sci 99:41-55

Gourdie RG, Severs NJ, Green CR, Rothery S, Germroth P, Thompson RP (1993) The spatial distribution and relative abundance of gap-junctional connexin40 and connexin43 correlate to functional properties of the cardiac atrioventricular conduction system. J Cell Sci 105:985-991

Gros D, Jarry-Guichard T, ten Velde I, De Mazière AMGL, Van Kempen MJA, Davoust J, Briand JP, Moorman AFM, Jongsma HJ (1994) Restricted distribution of connexin40, a gap junctional protein, in mammalian heart. Circ Res 74:839-851

Harris AL (2001) Emerging issues of connexin channels: biophysics fills the gap. Q Rev Biophys 34:325-472

Harris AL, Spray DC, Bennett MVL (1981) Kinetic properties of a voltage-dependent junctional conductance. J Gen Physiol 77:95117

He DS, Jiang JX, Taffet SM, Burt JM (1999) Formation of heteromeric gap junction channels by connexin 40 and 43 in vascular smooth muscle cells. Proc Natl Acad Sci USA 96:6495-6500

Hoyt RH, Cohen ML, Saffitz JE (1989) Distribution and threedimensional structure of intercellular junctions in canine myocardium. Circ Res 64:563-574

Kaba RA, Yacoub MH, Dupont E, Elneil S, Skepper J, Coppen SR, Pepper JR, Severs NJ (2003) Gap junctions in the atrio-ventricular conduction axis of the human heart. Eur Heart J 24:505

Kitamura H, Ohnishi Y, Yoshida A, Okajima K, Azumi H, Ishida A, Galeano EJ, Kubo S, Hayashi Y, Itoh H, Yokoyama M (2002) Heterogeneous loss of connexin43 protein in nonischemic dilated cardiomyopathy with ventricular tachycardia. J Cardiovasc Electrophysiol 13:865-870

Kléber AG, Rudy Y (2004) Basic mechanisms of cardiac impulse propagation and associated arrhythmias. Physiol Rev 84:431488 
Kléber AG, Janse MJ, Fast VG (2001) Normal and abnormal conduction in the heart. In: Handbook of Physiology, sect. 2, The Cardiovascular System, vol. 1, The Heart. Oxford: Oxford University Press, pp 455-530

Ko YS, Yeh HI, Ko YL, Hsu YC, Chen CF, Wu S, Lee YS, Severs NJ (2004) Three-dimensional reconstruction of the rabbit atrioventricular conduction axis by combining histological, desmin, and connexin mapping data. Circulation 109:1172-1179

Kostin S, Dammer S, Hein S, Klovekorn WP, Bauer EP, Schaper J (2004) Connexin 43 expression and distribution in compensated and decompensated cardiac hypertrophy in patients with aortic stenosis. Cardiovasc Res 62:426-436

Kostin S, Rieger M, Dammer S, Hein S, Richter M, Klovekorn WP, Bauer EP, Schaper J (2003) Gap junction remodeling and altered connexin43 expression in the failing human heart. Mol Cell Biochem 242:135-144

Kreuzberg MM, Schrickel JW, Ghanem A, Kim JS, Degen J, JanssenBienhold U, Lewalter T, Tiemann K, Willecke K (2006) Connexin30.2 containing gap junction channels decelerate impulse propagation through the atrioventricular node. Proc Natl Acad Sci USA 103:5959-5964

Loewenstein WR (1981) Junctional intercellular communication: the cell-to-cell membrane channel. Physiol Rev 61:829-913

Loewenstein WR, Socolar SJ, Higashino S, Kanno Y, Davidson N (1965) Intercellular communication: renal, urinary bladder, sensory, and salivary gland cells. Science 149:295-298

Martinez AD, Hayrapetyan V, Moreno AP, Beyer EC (2002) Connexin43 and connexin45 form heteromeric gap junction channels in which individual components determine permeability and regulation. Circ Res 90:1100-1107

Miquerol L, Meysen S, Mangoni M, Bois P, van Rijen HV, Abran P, Jongsma HJ, Nargeot J, Gros D (2004) Architectural and functional asymmetry of the His-Purkinje system of the murine heart. Cardiovasc Res 63:77-86

Moreno AP, Laing JG, Beyer EC, Spray DC (1995) Properties of gap junction channels formed of connexin 45 endogenously expressed in human hepatoma (SKHep1) cells. Am J Physiol 268:C356-C365

Peters NS, Green CR, Poole-Wilson PA, Severs NJ (1993) Reduced content of connexin43 gap junctions in ventricular myocardium from hypertrophied and ischaemic human hearts. Circulation $88: 864-875$

Polontchouk LD, Valiunas V, Haefliger J-A, Eppenberger HM, Weingart R (2002) Expression and regulation of connexins in cultured ventricular myocytes isolated from adult rat hearts. Pfluegers Arch 443:676-689

Rackauskas M, Kreuzberg MM, Pranevicius M, Willecke K, Verselis VK, Bukauskas FF (2007) Gating properties of heterotypic gap junction channels formed of connexins 40, 43 and 45. Biophys J 92:1952-1965

Severs NJ (1989a) Constituent cells of the heart and isolated cell models in cardiovascular research. In: Piper HM, Isenberg G (eds), Isolated Adult Cardiomyocytes, vol. 1. Boca Raton, FL: CRC Press, pp 3-41

Severs NJ (1989b) Gap junction shape and orientation at the cardiac intercalated disk. Circ Res 65:1458-1461

Severs NJ (1990) The cardiac gap junction and intercalated disc. Int J Cardiol 26:137-173

Severs NJ, Rothery S, Dupont E, Coppen SR, Yeh HI, Ko YS, Matsushita T, Kaba R, Halliday D (2001) Immunocytochemical analysis of connexin expression in the healthy and diseased cardiovascular system. Microsc Res Tech 52:301-322

Severs NJ, Coppen SR, Dupont E, Yeh HI, Ko YS, Matsushita T (2004a) Gap junction alterations in human cardiac disease. Cardiovasc Res 62:368-377
Severs NJ, Dupont E, Coppen SR, Halliday D, Inett E, Baylis D, Rothery S (2004b) Remodelling of gap junctions and connexin expression in heart disease. Biochim Biophys Acta 1662:138 148

Severs NJ, Dupont E, Kaba RA, Thomas N (2005) Gap junction and connexin remodeling in human heart disease. In: Winterhager $\mathrm{E}$ (ed), Gap Junctions in Development and Disease. Berlin: Springer, pp 57-82

Severs NJ, Dupont E, Thomas N, Kaba R, Rothery S, Jain R, Sharpey $\mathrm{K}$, Fry CH (2006) Alterations in cardiac connexin expression in cardiomyopathies. Adv Cardiol 42:228-242

Smith JH, Green CR, Peters NS, Rothery S, Severs NJ (1991) Altered patterns of gap junction distribution in ischemic heart disease. An immunohistochemical study of human myocardium using laser scanning confocal microscopy. Am J Pathol 139:801-821

Valiunas V (2002) Biophysical properties of connexin-45 gap junction hemichannels studied in vertebrate cells. J Gen Physiol 119:147-164

Valiunas V, Weingart R (2000) Electrical properties of gap junction hemichannels identified in transfected HeLa cells. Pfluegers Arch 440:366-379

Valiunas V, Bukauskas FF, Weingart R (1997) Conductances and selective permeability of connexin43 gap junction channels examined in neonatal rat heart cells. Circ Res 80:708-719

Valiunas V, Weingart R, Brink PR (2000) Formation of heterotypic gap junction channels by connexins 40 and 43 . Circ Res $86: \mathrm{e} 42$ e49

Valiunas V, Gemel J, Brink PR, Beyer EC (2001) Gap junction channels formed by coexpressed connexin 40 and connexin 43 . Am J Physiol 281:H1675-H1689

Van Kempen MJA, ten Velde I, Wessels A, Oosthoek PW, Gros D, Jongsma HJ, Moorman AFM, Lamers WH (1995) Differential connexin distribution accommodates cardiac function in different species. Microsc Res Tech 31:420-436

Van Rijen HVM, Wilders R, Van Ginneken ACG, Jongsma HJ (1998) Quantitative analysis of dual whole-cell voltage-clamp determinations of gap junction conductance. Pfluegers Arch 436:141151

Van Veen TA, van Rijen HV, Jongsma HJ (2000) Electrical conductance of mouse connexin45 gap junction channels is modulated by phosphorylation. Cardiovasc Res 46:496-510

Veenstra RD (1990) Voltage-dependent gating of gap junction channels in embryonic chick ventricular rat heart cells. Am J Physiol 258:C662-C672

Vogel R, Weingart R (1998) Mathematical model of vertebrate gap junctions derived from electrical measurements on homotypic and heterotypic channels. J Physiol 510.1:177-189

Vogel R, Weingart R (2002) The electrophysiology of gap junctions and gap junction channels and their mathematical modelling. Biol Cell 94:501-510

Vozzi C, Dupont E, Coppen SR, Yeh HI, Severs NJ (1999) Chamberrelated differences in connexin expression in the human heart. $\mathrm{J}$ Mol Cell Cardiol 31:991-1003

Wang HZ, Li J, Lemanski LF, Veenstra RD (1992) Gating of mammalian cardiac gap junction channels by transjunctional voltage. Biophys J 63:139-151

Weidmann S (1952) The electrical constants of Purkinje fibres. J Physiol 118:348-360

Weidmann S (1966) The diffusion of radiopotassium across intercalated disks of mammalian cardiac muscle. J Physiol 187:323-342

Weidmann S (1967) Cardiac electrophysiology in the light of recent morphological findings. Harvey Lect 61:1-15

Weingart R, Desplantez T, Thomas N, Severs NJ, Dupont E (2006) Cardiac gap junctions: properties of heteromeric channels probing connexin-tandem constructs. Proceedings of the ab- 
stracts of the International Conference on Physiological and Pathological Importance of Gap Junctions, Tokyo, Japan, November 20-22, 2006, p 26

Willecke K, Eiberger J, Degen J, Eckhardt D, Romualdi A, Güldenagel M, Deutsch U, Söhl G (2002) Structural and functional diversity of connexin genes in the mouse and human genome. Biol Chem 383:725-737

Yamada KA, Rogers JG, Sundset R, Steinberg TH, Saffitz JE (2003) Up-regulation of connexin45 in heart failure. J Cardiovasc Electrophysiol 14:1205-1212 